\title{
Acknowledgement to Reviewers of Remote Sensing in 2018
}

Remote Sensing Editorial Office

MDPI, St. Alban-Anlage 66, 4052 Basel, Switzerland

Published: 11 January 2019

Rigorous peer-review is the corner-stone of high-quality academic publishing. The editorial team greatly appreciates the reviewers who contributed their knowledge and expertise to the journal's editorial process over the past 12 months. In 2018, a total of 2044 papers were published in the journal, with a median time to first decision of 21 days and a median time to publication of 48 days. The editors would like to express their sincere gratitude to the following reviewers for their cooperation and dedication in 2018:

Aanstoos, James

Aaron, David

Abbas, Sawaid

Abboud, Marie

Abdel-Hamid, Ayman

Abd-Elrahman, Amr

Abdel-Rahman, Elfatih

Abdollahnejad, Azadeh

Abe, Takahiro

Abellan, Antonio

Abileah, Ron

Aboutalebi, Mahyar

Abrams, Michael

Abuzar, Mohammad

Acharya, Tri Dev

Acito, Nicola

Ackleson, Steve

Acquah, Gifty

Adami, Marcos

Adamo, Maria

Adán, Antonio

Adão, Telmo

Addink, Elisabeth

Adhikari, Hari

Adhikari, Loknath

Adler-Golden, Steven

Adriaensen, Stefan
Adriano, Bruno

Afrasinei, Gabriela Mihaela

Agapiou, Athos

Agathos, Alexander

Aggarwal, Hemant

Aghababaee, Hossein

Agrafiotis, Panagiotis

Agrillo, Emiliano

Aguado, Inmaculada

Agüera Vega, Francisco

Aguilera, Cristhia

Aguirre-Salado, Carlos

Ahmad, Tanveer

Ahmadalipour, Ali

Ahmed, M. Razu

Ahmed, Mohamed

Ahmed, Samir

Aina, Yusuf Adedoyin

Ainouz, Samia

Ainsworth, Thomas

Ajadi, Olaniyi

Akbar, Muhammad

Akbar, Ruzbeh

Akca, Devrim

Akhtar, Naveed

Akihiko, Kuze

Akkari, Cherine 
Akumaga, Uvirkaa

Akusok, Anton

Alados, Concepcion

Alaghmand, Sina

Alam, Mushfiqul

Alappattu, Denny

Albano, Matteo

Albergel, Clement

Alberti, Giovanni

Albino, Fabien

Albukhanajer, Wissam

Alcântara, Enner

Aldrich, Stephen

Alemohammad, Seyed Hamed

Alexakis, Dimitrios

Alexander, Cici

Alexandri, Georgia

Alexandridis, Thomas

Alganci, Ugur

AlHichri, Haikel

Ali, Abebe M

Aliahmad, Behzad

Aljaf, Arsalan

Alkan, Reha Metin

Allan, Richard

Alleaume, Samuel

Allen, George

Allen, Michael

Almeida, Luis Pedro

Alonso, Luis

Alonso-Benito, Alfonso

Alparone, Luciano

Alpers, Werner

Alqurashi, Abdullah

Al-Rawabdeh, Abdullah

Alsadik, Bashar

Alsina-Pagès, Rosa Ma

Alsweiss, Suleiman

Al-Tahir, Raid

Altena, Bas

Álvarez Fernández, Martina Inmaculada

Alvarez Lopez, Yuri

Álvarez, Orlando
Alvioli, Massimiliano

Al-Yaari, Amen

Amaral, Cibele

Amato, Federico

Amato, Umberto

Amatulli, Giuseppe

Amatya, Suraj

Ambrosia, Vincent

Amezcua, Javier

Amitrano, Donato

An, Nan

Anagnostou, Marios

Anaya, Jesús

Ancuti, Cosmin

Anderson, Christopher

Anderson, Ray

Anderson, Sharolyn

Anderson, Stuart

Andersson, Magnus

Andreo, Veronica

Andronache, Ion

Aneece, Itiya

Angelini, Maria Giuseppa

Angelliaume, Sebastien

Anghel, Andrei

Anstee, Janet

Antal, Hiba

Anterrieu, Eric

Anthes, Richard

Antonarakis, Alexander

Antonelli, Paolo

Antonio Valiente, Jose

Antropov, Oleg

Anweiler, Stanisław

Aoki, Yosuke

Aplin, Paul

Araki, Hiroshi

Aralova, Dildora

Aranha, Jose

Arbelo, Manuel

Ardizzone, Francesca

Ardö, Jonas

Aretxabaleta, Alfredo 
Arguedas, Virginia Fernandez

Argüello, Francisco

Arief, Hasan Asy'ari

Arikan, Feza

Arjasakusuma, Sanjiwana

Armstrong, Roy

Armstrong, William

Arndt, Jan Erik

Arrogante-Funes, Patricia

Arroyo-Mora, Juan Pablo

Artese, Giuseppe

Aryal, Jagannath

Arza, Marcos

Asgary, Ali

Ashby, Jeffrey

Askne, Jan

Aslan, Aslan

Asong, Zilefac Elvis

Aspandiar, Mehrooz

Asplin, Matthew

Assal, Timothy

Atherton, Jon

Atkins, Jeff

Attila, Jenni

Atzberger, Clement

Aulicino, Giuseppe

Avellar, Gustavo

Avtar, Ram

Awad, Mohamad

Azadbakht, Mohsen

Azzoni, Roberto Sergio

B. Da Silva, Bernardo

Baade, Jussi

Baba, Wassim Mohamed

Babaeian, Ebrahim

Bach, Heike

Bachmann, Martin

Bachmann, Roger

Bachofer, Felix

Ba,czyk, Marcin Kamil

Bader, Brett

Badescu, Gabriel

Badulin, Sergei
Baghdadi, Nicolas

Baguis, Pierre

Bahadur Thapa, Rajesh

Bai, Hua

Bainbridge, Scott

Baines, Peter G.

Baiocchi, Valerio

Baird, Mark

Bajgain, Rajen

Bajocco, Sofia

Baker, David

Baker, Robert

Bakker, Wim

Bakuła, Krzysztof

Bakuła, Mieczysław

Balachandran, Sivaraman

Balali, Vahid

Balasubramanian, Sundarabalan

Balázs, Péter

Balenović, Ivan

Ball, John

Ballester, Carlos

Balletti, Caterina

Balouin, Yann

Balsa-Barreiro, Jose

Balsi, Marco

Balss, Ulrich

Baltazart, Vincent

Balz, Timo

Banda, Juan

Banerjee, Ruman

Bange, Jens

Bannari, Abderrazak

Baraković Husić, Jasmina

Baran, Remigiusz

Baray, Jean-Luc

Barba, Salvatore

Barbosa, Ana Branco

Barbosa, Henrique

Barfus, Klemens

Barkat, Adnan

Barker, Michael Kenneth

Barletta, Valentina 


\begin{tabular}{|c|c|}
\hline Barnaba, Francesca & Belgiu, Mariana \\
\hline Barnes, Brian & Belikov, Dmitry \\
\hline Barone, Pier Matteo & Bell, David \\
\hline Barr, Iestyn & Bellacicco, Marco \\
\hline Barrett, Brian & Bellasio, Roberto \\
\hline Barriot, Jean-Pierre & Belletti, Barbara \\
\hline Barsanti, Sara Gonizzi & Bellos, Vasilis \\
\hline Barsi, Arpad & Belyaev, Evgeny \\
\hline Bartholomeus, Harm & Bendig, Juliane \\
\hline Bartolo, Renee & Bendixen, Mette \\
\hline Baryla, Radoslaw & Ben-Moshe, Boaz \\
\hline Barzaghi, Riccardo & Bennett, Mia M \\
\hline Bas, Joan & Bennett, Michael \\
\hline Bascietto, Marco & Bentel, Katrin \\
\hline Bašić, Tomislav & Bentes, Carlos \\
\hline Basler, David & Bento, Virgílio \\
\hline Bass, Leonid & Berezowski, Tomasz Edward \\
\hline Bassani, Cristiana & Bergeot, Nicolas \\
\hline Bastos, Ana & Berger, Katja \\
\hline Basu, Saikat & Bergillos, Rafael \\
\hline Batar, Amit & Berglund, Robin \\
\hline Bathrellos, George & Bergstedt, Helena \\
\hline Battaglia, Alessandro & Berisha, Sebastian \\
\hline Baumann, Sabine & Berland, Adam \\
\hline Bayissa, Yared & Bernabe Garcia, Sergio \\
\hline Baynard, Christopher & Bernier, Monique \\
\hline Bazi, Yakoub & Beron-Vera, Francisco \\
\hline Bazzino Ferreri, Gaston & Berry, David \\
\hline Beamish, Alison & Bertone, Edoardo \\
\hline Becek, Kazimierz & Berveglieri, Adilson \\
\hline Bech, Joan & Bettenhausen, Michael \\
\hline Bechtel, Benjamin & Bettinger, Pete \\
\hline Bechtold, Michel & Betts, Alan \\
\hline Beck, John & Beyerer, Jürgen \\
\hline Beck, Richard Allan & Beylich, Achim \\
\hline Beckers, Joost & Bhandari, Santosh \\
\hline Beckett, Keith & Bhardwaj, Anshuman \\
\hline Beeri, Ofer & Bhatt, Rajendra \\
\hline Begue, Agnes & Bhattarai, Keshav \\
\hline Beighley, Edward & Bhattarai, Nishan \\
\hline Beisl, Ulrich & Bi, Jian \\
\hline Belagiannis, Vasileios & Bialik, Robert \\
\hline Bélair, Stéphane & Biancamaria, Sylvain \\
\hline
\end{tabular}


Bianchi, Tiziano

Bidel, Luc

Biggs, Trent

Bignami, Christian

Bignami, Francesco

Bijker, Wietske

Bilal, Muhammad

Bilbao, Julia

Billi, Andrea

Bilotta, Giuliana

Bingham, Fred

Binietoglou, Ioannis

Biondi, Filippo

Biondi, Riccardo

Biro, Khaled

Bishop, Michael

Biudes, Marcelo Sacardi

Bizzi, Simone

Blackett, Matthew

Blanco-Jarvio, Anidia

Blankenship, Clay

Błaszczak-Bąk, Wioleta

Bleiweiss, Max

Blenkinsopp, Chris

Blesius, Leonhard

Blevins, Robert

Bliss, Angela

Blomberg, Ann Elisabeth Albright

Blonski, Slawomir

Bobojonov, Ihtiyor

Böck, Sebastian

Bocklitz, Thomas

Bodoque, José

Boergens, Eva

Bogdan, Ileana

Bohling, Björn

Bohm, Emanuele

Boisvert, Linette

Bojariu, Roxana

Bolten, Andreas

Bolvin, David

Bonafoni, Stefania

Bonenberg, Lukasz
Bonfil, David

Bonì, Roberta

Bonin, Timothy

Bonin-Font, Francisco

Bookhagen, Bodo

Borak, Jordan

Bordogna, Gloria

Borghi, Alessandra

Borkowski, Andrzej

Borkowski, Piotr

Börner, Anko

Borries, Claudia

Bos, Machiel

Bosch, Xavi

Boschetti, Luigi

Boschetti, Mirco

Boscutti, Francesco

Botha, Elizabeth Johanna

Bouaziz, Moncef

Boulet, Gilles

Boutin, Jacqueline

Bouwmans, Thierry

Bovenga, Fabio

Boy, Jean-Paul

Boyd, Doreen

Bozzano, Francesca

Bradley, Patrick Erik

Brajard, Julien

Bramstedt, Klaus

Brancato, Virginia

Brandini, Carlo

Brando, Vittorio

Brasebin, Mickael

Braun, Alexander

Brazel, Anthony

Brede, Benjamin

Breili, Kristian

Bresciani, Mariano

Breunig, Fabio Marcelo

Brian, Hirth

Bright, Ben

Bringi, Viswanathan

Brinkhoff, James 


\begin{tabular}{|c|c|}
\hline Brinkmann, Katja & Burud, Ingunn \\
\hline Brisco, Brian & Butt, Jemil Avers \\
\hline Brivio, Pietro Alessandro & Buttafuoco, Gabriele \\
\hline Brocca, Luca & Buza, Krisztian \\
\hline Brockmann, Carsten & Byon, Young-Ji \\
\hline Broerse, Тaco & Bystrov, Aleksandr \\
\hline Brolly, Matthew & Caballer, Jaime Almonacid \\
\hline Brooks, Evan & Cabboi, Alessandro \\
\hline Brotas, Vanda & Cabo, Carlos \\
\hline Brovelli, Maria Antonia & Cabot, Francois \\
\hline Browell, Jethro & Cabrera, Humberto \\
\hline Brown, Dana & Cachorro, Victoria \\
\hline BROWN, Ian & Cadeddu, Maria \\
\hline Brown, Michael & Cahalane, Conor \\
\hline Brown, Molly & Cai, Xiaohao \\
\hline Broxton, Patrick & Calbó, Josep \\
\hline Bruguera, Antoni & Calcaterra, Domenico \\
\hline Brühl, Christoph & Calderhead, Angus Ian \\
\hline Bruna, Arcangelo & Calders, Kim \\
\hline Brunn, Andreas & Caldwell, Todd \\
\hline Bruno, Maria Francesca & Calera, Alfonso \\
\hline Brusaporci, Stefano & Calheiros, Alan \\
\hline Bryan, Sean & Callahan, Philip \\
\hline Bryla, David & Calleja, Javier Fernández \\
\hline $\mathrm{Bu}$, Honggang & Callico, Gustavo \\
\hline Buchholz, Rebecca & Calò, Fabiana \\
\hline Buchroithner, Manfred & Calvari, Sonia \\
\hline Buddenbaum, Henning & Calveras, Jordi Llorens \\
\hline Budge, Scott & Calvet, Jean-Christophe \\
\hline Budillon, Alessandra & Camacho, Fernando \\
\hline Bueno, Gloria & Cammalleri, Carmelo \\
\hline Buitenwerf, Robert & Campillo, Carlos \\
\hline Bukin, Oleg & Campos, Néstor \\
\hline Bulgin, Claire & Campos-Taberner, Manuel \\
\hline Buller, William & Camps, Adriano \\
\hline Bunting, Erin & Campus, Paola \\
\hline Buono, Andrea & Candiani, Gabriele \\
\hline Burai, Péter & Cantoro, Gianluca \\
\hline Burakowski, Elizabeth & Cao, Changyong \\
\hline Burbey, Thomas & Cao, Kai \\
\hline Burgdorf, Martin & Cao, Lin \\
\hline Burgdorf, Martin Jörg & Cao, Ning \\
\hline Burian, Adina & Capobianco, Giuseppe \\
\hline
\end{tabular}


Capodici, Fulvio

Capolupo, Alessandra

Caporali, Alessandro

Capozzoli, Luigi

Capuano, Vincenzo

Carabez, Eduardo

Cardellach, Estel

Carey, Lawrence

Carfora, Maria Francesca

Cariou, Claude

Carlson, Daniel Frazier

Carlson, Toby

Carmon, Nimrod

Carmona-Moreno, Cesar

Caro-Cuenca, Miguel

Carr, Brett

Carrasco, Luis

Carrasco, Ruben

Carrera, Marco

Carroll, Mark

Carter, William

Cartus, Oliver

Casadio, Stefano

Casaseca, Pablo

Caselles, Vicente

Castaldo, Paolo

Castaldo, Raffaele

Castañeda, Carmen

Castellani, Beatrice

Castellani, Francesco

Castellazzi, Pascal

Castelli, Elisa

Casterad, Ma Auxiliadora

Castilla, Guillermo

Castillo, Jose Alan

Castro, Ana I.de

Casula, Giuseppe

Catalin Petritan, Ion

Catry, Thibault

Cattani, Elsa

Caturegli, Lisa

Caughlin, T Trevor

Cavaleri, Luigi
Cavalié, Olivier

Cavalli, Marco

Cavalli, Rosa

Cavalli, Rosa Maria

Cawkwell, Fiona

Caya, Alain

Cazacu, Marius Mihai

Cazzaniga, Noemi

Cecinati, Francesca

Centeno, Jorge Antonio Silva

Cerasoli, Sofia

Cerdà, Artemi

Cermak, Jan

Cerra, Daniele

Cesaroni, Claudio

Cetin, Mufit

Ch. Miliaresis, George

Chadwick, William

Chakraborty, Sumit

Chamorro, Leonardo

Champagne, Catherine

Champion, Nicolas

Champollion, Nicolas

Chan, Kam Wai Clifford

Chan, Pak-Wai

Chan, Steven

Chang, Hsiao-Tung

Chang, Jiyul

Chang, Kuo-Jen

Chang, Liling

Chang, Michael

Chan-Hon-Tong, Adrien

Chanover, Nancy

Chao, Benjamin

Chaplot, Vincent

Chapman, Bruce

Chapron, Bertrand

Charou, Eleni

Chase, Alison

Chase, Arlen

Chau, Kwok-wing

Chavanne, Cédric

Chazette, Patrick 
Checa-Garcia, Ramiro

Chemura, Abel

Chen, Assaf

Chen, Bin

Chen, Cao

Chen, Chen

Chen, Chi

Chen, Chien-yuan

Chen, Fulong

Chen, Haonan

Chen, Jiquan

Chen, Kejie

Chen, Kongzhe

Chen, Kun-Shan

Chen, Liang

Chen, Luzhen

Chen, Maosi

Chen, Qi

Chen, Wei-Nai

Chen, Xianfeng

Chen, Xiuhong

Chen, Yuehong

Chen, Yu-Hsuan

Chen, ZhiQiang

Chen, Zhongbing

Cheng, Gong

Cheng, Jie

Cheng, Ke-Sheng

Cheng, Tao

Cheng, Yongcun

Chennu, Arjun

Chernetskiy, Maxim

Chernyi, Sergei

Cherukuru, Nihanth

Chevallier, Frédéric

Chew, Clara

Cheynet, Etienne

Chhetri, Parveen Kumar

Chi, Junhwa

Chiabrando, Filiberto

Chickadel, Chris

Chien, Hwa

Chiesi, Marta
Chini, Marco

Chio, Shih-Hong

Chipman, Jonathan

Chirici, Gherardo

Chisholm, Sallie

Chishtie, Farrukh

Chiu, Long $S$

Cho, Byoung-Kwan

Cho, Eunsang

Cho, Hyun Jung

Cho, Jaeil

Cho, Kyung Hwa

Cho, Younghyun

Chodak, Marcin

Choi, Jaewan

Choi, Kyoungah

Choi, Minha

Choi, Myungje

Choi, Sungho

Chou, Jung Hua

Chou, Tien-Yin

Chow, Jacky

Chretien, Stephane

Christaras, Basile

Christiansen, Martin Peter

Christie, Frazer

Christman, Zachary

Chrysos, Michael

Chu, Thuan

Chuang, Yung-Chung

Chum, Jaroslav

Chung, Eui-Seok

Churnside, James

Chybicki, Andrzej

Ciabatta, Luca

Ciaccheri, Leonardo

Ciampalini, Andrea

Ciampitti, Ignacio Antonio

Ciancia, Emanuele

Ciecholewski, Marcin

Cięszczyk, Sławomir

Cifelli, Robert

Cigna, Francesca 
Ciolli, Marco

Ciraolo, Giuseppe

Ciren, Pubu

Ciro, Manzo

Citterio, Michele

Clark Sutterley, Tyler

Clarke, Keith

Claverie, Martin

Cleguer, Christophe

Clerbaux, Nicolas

Clevers, Jan

Clive Banks, Andrew

Cloern, Jim

Cloutis, Edward

Coburn, Craig

Cohen, Jason Blake

Cointault, Frédéric

Colin Koeniguer, Elise

Colliander, Andreas

Collins, Michael

Colom, Miguel

Colombo, Claudio

Colomina, Ismael

Colpaert, Alfred

Coluzzi, Rosa

Comba, Lorenzo

Comer, Douglas

Comite, Davide

Condessa, Filipe

Conforti, Massimo

Confuorto, Pierluigi

Congalton, Russel

Constantin, Sorin

Contini, Daniele

Contreras-Medina, Luis

Convertino, Matteo

Conyers, Lawrence

Cook, Alison

Cooper, Matthew

Coopersmith, Evan

Corbane, Christina

Corbella, Ignasi

Corbelle Rico, Eduardo Jose
Corbí, Hugo

Corchado Rodríguez, Juan Manuel

Corcoran, Katie

Cordon, Gabriela

Corgnati, Lorenzo

Corney, Stuart

Corrales Ramon, Juan Antonio

Corstanje, Ronald

Coscieme, Luca

Cosentino, Antonino

Cosoli, Simone

Costa, Gilson

Costa, Hugo

Costa, J. Miguel

Costabile, Pierfranco

Costafreda-Aumedes, Sergi

Costanzo, Antonio

Côté, Stéphane

Coudert, Benoit

Couteron, Pierre

Coutinho, Alexandre

Cowley, Dave

Cox, Christopher

Coy, André

Cozzolino, Daniel

Cozzolino, Davide

Crago, Richard

Crespi, Mattia

Crisman, Thomas

Cristóbal Rosselló, Jordi

Crommelinck, Sophie

Crosetto, Michele

Crosman, Erik

Crosson, William

Crout, Richard

Crow, Wade

Cruise, James

Cruz, Henry

Csillik, Ovidiu

Cudahy, Thomas

Cuenca-Garcia, Carmen

Cuesta-Frau, David

Cui, Minshan 
Cui, Song

Cummings, Anthony

Cunha, Mario

Cura, Rémi

Currey, Chris

Custódio, Danilo

Czarnogorska, Magdalena

D'Andrimont, Raphaël

D'Urso, Guido

Daanen, Ronald

Dąbek, Paweł

Dabove, Paolo

Dabrowski, Pawel

Dąbski, Maciej

DaCamara, Carlos

D'Acci, Luca

D'Addabbo, Annarita

D'Adderio, Leo Pio

Dahlin, Kyla

Dahmane, Mohamed

Dai, Keren

Dal Sasso, Silvano

Dalla Corte, Ana Paula

Dalla Mora Esquerdo, Julio Cesar

Dalponte, Michele

Daly, Karen

Dandois, Jonathan

Daniel, Jones

Danilo, Céline

Dannowski, Ralf

Dapo, Almin

Darbeheshti, Neda

Dardanelli, Gino

Darsena, Donatella

Dartnell, Peter

Dash, Jonathan

Davis, W. Jackson

Dawidowicz, Karol

Day, Terence

De Angelis, Guido

De Blasio, Gabriel

De Bruin, Sytze

De Donato, Philippe
De Filippo, Daniela

De Freitas, Edison Pignaton

De Gaetani, Carlo

De Jong, Wil

De Kloe, Jos

De Kok, Roeland

De Luca, Davide Luciano

De Meo, Pasquale

De Miguel, Argimiro

De Miguel, Gonzalo

De Novellis, Vincenzo

De Pablo, M.A.

De Rydt, Jan

De Valck, Jeremy

De Vries, Walter

De Zan, Francesco

Deb, Proloy

Declercq, Pierre-Yves

Defraigne, Pascale

Dehghanian, Vahid

Dehn, Jonathan

Deilami, Kaveh

Dekker, Arnold

Del Negro, Ciro

Del Soldato, Matteo

Delalieux, Stephanie

Delaney, Cathy

Delaney, Keith

Delgado, Jorge

Delle Donne, Dario

Demarq, Hervé

Demetriou, Elias

Demir, Nusret

Demirel, Mehmet

Dempewolf, Jan

Denbina, Michael

Deng, Chengbin

Deng, Xiaoli

Deng, Yingbin

Deng, Zhiguo

Deniziak, Stanisław

Dennison, Philip

Deo, Ram Kumar 
Deprem, Zeynel

Deshpande, Sagar

Dettmering, Denise

Deutscher, Janik

Dev, Soumyabrata

Devaraju, Balaji

Devoti, Roberto

Devred, Emmanuel

DeVries, Ben

Dewan, Ashraf

Dewi, Ratna Sari

Dewitte, Steven

Dherbecourt, Jean-Baptiste

Dhungel, Ramesh

Di Biagio, Claudia

Di Bisceglie, Maurizio

Di Cicco, Annalisa

Di Martino, Gerardo

Di Martire, Diego

Di Paola, Francesco

Di Simone, Alessio

Di Traglia, Federico

Dial, Roman

Diani, Marco

Díaz, Felipe

Diaz, Maria

Diaz, Oliver

Diaz-Barradas, Mari Cruz

Dietz, Andreas

Dietze, Michael

Dietzsch, Felix

Dilts, Tom

DiMarchi, Massimo

Dimuccio, Luca

Dinardo, Salvatore

Dinelli, Bianca Maria

Ding, Jiachen

Ding, Yongjian

Dippner, Joachim

Disperati, Leonardo

Djebou, Dagbegnon C. Sohoulande

Dobinski, Wojciech

Dobler, Gregory
Dobreva, Iliyana

Doctor, Katarina

Doelling, Dave

Doglioni, Angelo

Dogliotti, Ana Ines

Dokladal, Petr

Domingues Franceschini, Marston Héracles

Dominici, Donatella

Donchyts, Gennadii

Doneus, Michael

Dong, Chun-Yu

Dong, Di

Dong, Jianzhi

Dong, Jinwei

Dong, Kim

Dong, Taifeng

Dong, Zhen

D'Oreye, Nicolas

Dou, Jie

Doubrawa, Paula

Doulamis, Anastasios

Doulamis, Nikolaos

Dragozi, Eleni

Drake, Jason

Drake, Nick

Drap, Pierre

Drazen, David

Dreyer, Marion

Drolet, Guillaume

Dronova, Iryna

Drumetz, Lucas

Du, Jinyang

Duan, Sibo

Duarte, Diogo

Duarte-Carvajalino, Julio

Dube, Timothy

Dubey, Harishchandra

Dudczyk, Janusz

Duguay, Yannick

Đuka, Andreja

Duma, Virgil-Florin

Dumic, Emil

Dumitrescu, Alexandru 
Dumont, Stéphanie

Dunai, Larisa

Duncan, David

Duncanson, Laura

Dunea, Daniel

Durack, Paul

D'Urso, Guido

Dvornikov, Yury

Dwomoh, Francis

Dworak, Volker

Dwyer, John

Dybkjær, Gorm

Dyer, Jamie

Dykema, John

Dyson, Jack

Eastman, Roger

Ebuchi, Naoto

Echevin, Vincent

Ediriweera, Sisira

Edwards, Andrew

Eekhout, Joris

Efremenko, Dmitry

Egido, Alejandro

Eineder, Michael

Einzmann, Kathrin

Eisank, Clemens

Eitzen, Zachary

Eklund, Lina

Elaksher, Ahmed

El-Askary, Hesham

Elberink, Sander Oude

Eldhuset, Knut

Elefante, Stefano

Elfadaly, Abdelaziz

El-Hajj, Mohamed

Elias, Panagiotis

Elsaesser, Gregory

Elsner, Paul

Elvidge, Chris

Emeis, Stefan

Emmer, Adam

Enderlin, Ellyn

Ene, Liviu Theodor
Engelbrecht, Jeanine

Engman, Edwin

Engstrom, Ryan

Enguita, José María

Enríquez, Susana

Eo, YangDam

Erban, Laura

Ercanoglu, Murat

Eren, Firat

Eresmaa, Reima

Erinjery, Joseph

Erives, Hector

Ermida, Sofia

Erten, Esra

Escorihuela, María José

Estornell, Javier

Evans, Bradley John

Evans, Ian

Even, Markus

Ewans, Kevin

Ewijk, Karin Van

Eyles, Nick

Eymard, Laurence

Fabre, Sophie

Fagin, Todd

Fai A. Lo, Kwong

Fairley, Iain

Faisal, Kamil

Faivre, Robin

Fakiris, Elias

Falco, Nicola

Falco, Pierpaolo

Falcone, James

Falk, Ulrike

Fan, Fernando

Fan, Jinlong

Fan, Lei

Fan, Xingwang

Fang, Hongliang

Fang, Peng

Fanti, Riccardo

Faour, Ghaleb

Farahmand, Alireza 
Farolfi, Gregorio

Farquharson, Jamie

Farquharson, Louise

Farr, Thomas

Farr, Tom

Faruolo, Mariapia

fassnacht, fabian

Fatoyinbo, Temilola

Fatras, Christophe

Fava, Francesco Pietro

Favali, Paolo

Fayaz, Mohammadreza

Fedeli, Alessandro

Federico, Ivan

Feige, Kathrin

Feist, Dietrich

Felisberto, Paulo

Femiani, John

Fencik, Robert

Feng, Hui

Feng, Min

Feng, Wanpeng

Feng, Xingru

Fenoglio-Marc, Luciana

Feofilov, Artem

Fera, Martin

Ferecatu, Marin

Feret, Jean Baptiste

Fernandes, Francisco

Fernandes, Joana

Fernandes, João Catalão

Fernandes, Paulo

Fernandes, Richard

Fernández Jaramillo, Arturo Alfonso

Fernández, Alberto

Fernández, Tomás

Ferrando, Ilaria

Ferrari, Marco

Ferraz, Antonio

Ferreira, Hugo Almeida

Ferreira, Matheus Pinheiro

Ferreira, Vagner

Ferretti, Alessandro
Ferro-Famil, Laurent

Ferster, Brady

Fialko, Yuri

Fiaschi, Simone

Fickert, Thomas

Fidanova, Stefka

Fiedler, Brian

Fiedler, Emma

Figurski, Mariusz

Fikfak, Alenka

Filchev, Lachezar

Filippelli, Gabriel

Filipponi, Federico

Fingas, Merv

Finkensieper, Stephan

Fiorentino, Costanza

Fiorillo, Edoardo

Fiorucci, Federica

Fischer, Andrew

Fisher, Daniel

Fleming, Kevin

Fletcher, Andrew

Fletcher, Reginald

Fletcher, Steven

Floors, Rogier

Flores Tapia, Daniel

Flores, Gerardo

Flores, Juan

Flores-de Santiago, Francisco Javier

Floriano, Andrés García

Floricioiu, Dana

Floris, Mario

Fonseca, Ana

Fonte, Cidália Costa

Foody, Giles

Forkuor, Gerald

Forlani, Gianfranco

Formisano, Antonio

Förstner, Jochen

Fortunato, Eduardo

Foster, Michael

Foti, Dora

Foucher, Samuel 
Foucras, Myriam

Foumelis, Michael

Fournier, Richard

Fournier, Severine

Fox, Andrew Mark

Fox, Neil

Frabrizio, Santi

Fragoso Jr, Carlos Ruberto

Fraile, Roberto

Fraisse, Renaud

Francesca Carfora, Maria

Franci, Francesca

Francioni, Mirko

Franklin, Meredith

Franks, Sharon

Franz, Kristie

Franzen, Dave

Frappart, Frédéric

Fraser, Clive

Fraser, Robert $\mathrm{H}$

Frelich, Lee

Fretwell, Peter

Freund, Friedemann

Friedl, Peter

Friedrich, Katja

Friesen, John

Friess, Dan

Frodella, William

Frontoni, Emanuele

Frost, Anja

Fryskowska, Anna

$\mathrm{Fu}$, Jianshuang

$\mathrm{Fu}$, Lee-Lueng

$\mathrm{Fu}$, Peng

Fuckar, Neven

Fuhrmann, Thomas

Fujibe, Fumiaki

Fujimura, Atsushi

Fujisaki, Ikuko

Fujita, Mikiko

Fukushima, Norishige

Funatsu, Beatriz

Furnari, Antonino
Fusco, Adele

Fusco, Giannetta

Fuse, Takashi

Gabarda, Salvador

Gabella, Marco

Gabr, Safwat

Gabriel, Jose Luis

Gabrieli, Andrea

Gad, Sabreen

Gade, Martin

Gaetana, Ganci

Gaffney, Rowan

Gaiani, Marco

Gaidzik, Krzysztof

Gal, Martin

Gala, Tekleab

Gallant, Alisa

Gallay, Michal

Gallegos-Funes, Francisco

Galleguillos, Mauricio

Gallo, Kevin

Galvani, Alessandro

Galve, Joan Miquel

Galve, Jorge Pedro

Ganas, Athanassios

Gandois, Laure

Gangodagamage, Chandana

Gao, Bocai

Gao, Feng

Gao, Junfeng

Gao, Lianru

Gao, Meng

Gao, Qi

Gara, Tawanda

Garaba, Shungu

Garay, Michael

Garcia Tejero, Ivan Francisco

Garcia, David

Garcia, Pilar

Garcia, Ricardo

Garcia, Rodrigo

García-Cortés, Silverio

Garcia-Gutierrez, Jorge 
García-Olmo, Juan

Garcia-Pedrero, Angel

Garcia-Pineda, Oscar

García-Rigo, Alberto

Garcia-Ruiz, Francisco

Garcia-Santos, Vicente

Gardiner, Tom

Gärtner, Philipp

Garza, Rodrigo

Garzelli, Andrea

Garzón, Gracia Ester Martín

Gascoin, Simon

Gašparović, Mateo

Gastellu-Etchegorry, Jean Philippe

Gatebe, Charles

Gatis, Naomi

Gatziolis, Demetrios

Gaume, Eric

Gay, Michel

Ge, Cui

Ge, Maorong

Gege, Peter

Geiger, Alain

Geiss, Christian

Geldmann, Jonas

Geldsetzer, Torsten

Gelybó, Györgyi

Gencaga, Deniz

Genderen, John Van

Genet, Helene

Gennarelli, Gianluca

Genzano, Nicola

Georganos, Stefanos

George, Sony

Georgiadis, Charalampos

Georgiadis, Teodoro

Georgiev, Iliya

Georgoulias, Aristeidis

Gerard, France

Gerassimos, Peteinatos

Géraud, Thierry

Gerber, Florian

Gerhards, Max
Gerke, Markus

Gertner, George

Gevaert, Caroline

Ghafari, Elaheh

Ghaffari Razin, Mir Reza

Ghanmi, Helmi

Gharechelou, Saeid

Ghavidel, Ali

Gholizadeh, Asa

Ghoneim, Eman

Ghosh, Aniruddha

Ghosh, Tilottama

Ghuffar, Sajid

Gianelle, Damiano

Giannico, Vincenzo

Giannini, Fernanda

Gianniou, Michail

Giano, Salvatore Ivo

Gibbs, Ann

Gibson, John

Gienko, Gennady

Gierens, Klaus

Gignac, Charles

Gigović, Ljubomir

Gillespie, Thomas

Ginsca, Alexandru Lucian

Gioia, Andrea

Gioia, Ciro

Gioia, Dario

Gioli, Beniamino

Giordan, Daniele

Girelli, Valentina Alena

Girod, Luc

Gislum, René

Gitas, Ioannis

Giuliani, Gregory

Giulio Tonolo, Fabio

Gizachew, Belachew

Gjevestad, Jon Glenn

Gkemitzi, Alexandra

Gleich, Dusan

Glisan, Justin

Głomb, Przemysław 
Gnyp, Martin

Godah, Walyeldeen

Godone, Danilo

Goedemé, Toon

Goel, Salil

Goethals, Peter

Goetz, Jason

Gohar, Ghazaryan

Gohin, Francis

Gojda, Martin

Gokaraju, Balakrishna

Gökkaya, Kemal

Gokon, Hideomi

Golbon-Haghighi, Mohammad-Hossein

Goldberg, Daniel

Golston, Levi

Gomariz-Castillo, Francisco

Gomes, Eduardo

Gómez Doménech, Igor

Gomez, Cécile

Gomez, Cristina

Gomez, Luis

Gómez, Luis

Gomez-Chova, Luis

Gomez-Dans, Jose

Gómez-Enri, Jesús

Gomez-Ruiz, Jose Antonio

GOMMES, René Alex

Gomolka, Zbigniew

Gonçalves, Gil

Gonçalves, João

Gonçalves, Luísa M S

Goncalves, Olivier

Gonçalves, Paulo Jorge Sequeira

Gong, Wenlin

Gong, Wenyu

Goniewicz, Krzysztof

Gonome, Hiroki

Gonsamo, Alemu

Gonzalez Bonilla, Maria Jose

Gonzalez, Jose

Gonzalez, Rocio

González, Xesús
González-Dugo, Maria Pat

González-Gambau, Verónica

González-Hidalgo, Manuel

González-Rivero, Manuel

González-Zamora, Ángel

Gonzalo, Consuelo

Gonzalo-Martin, Consuelo

Goodbody, Tristan

Goodin, Douglas

Goodrich, Gregory

Goorts, Partrik

Goovaerts, Pierre

Gopinath, Girish

Górecki, Tomasz

Goretti, Agostino

Görgens, Eric B.

Gorroño, Javier

Gorte, Ben

Goshtasby, Arthur Ardeshir

Gottschall, Julia

Gouhier, Mathieu

Goulas, Yves

Gounaridis, Dimitris

Gouweleeuw, Ben

Gozdowski, Dariusz

Gradinaru, Simona

Graettinger, Alison

Graham, Robert

Gräler, Benedikt

Grams, Heather

Grant, Ian

Graw, Valerie

Gray, Josh

Graziani, Alberto

Graziano, Maria Daniela

Grebby, Stephen

Green, David

Green, Stuart

Greidanus, Harm

Grenzdörffer, Görres

Griggs, Erin

Grigillo, Dejan

Grigolato, Stefano 
Grigoras, Ion

Grigoriadis, Vassilios

Grimaldi, Salvatore

Grimaldi, Stefania

Grinham, Alistair

Grippa, Tais

Gristey, Jake

Grodsky, Semyon

Groh, Andreas

Groom, Geoffrey Brian

Gross, Barry

Große-Stoltenberg, André

Grote, Anne

Grote, Rüdiger

Grover, Aakriti

Gruber, Thomas

Gryning, Sven-Erik

$\mathrm{Gu}$, Huan

Gu, Zhu-Jun

Gualda, Javier Estévez

Guariglia, Emanuel

Guarnieri, Andrea Monti

Guccione, Pietro

Guenter, Frank

Guerci, Joseph

Guerra, Raúl

Guerra-Hernández, Juan

Guerrero-Rascado, Juan Luis

Guerriero, Leila

Guivant, Jose

Gultepe, Ismail

Gunn, Grant

Gunter, William Scott

Guo, Hong

Guo, Jiming

Guo, Xulin

Guo, Yi

Gurney, Robert

Gutiérrez-Tobal, Gonzalo

Gutjahr, Karlheinz

Guyet, Thomas

Guzman, Sandra

Gwak, Jeonghwan
Gwenzi, David

Haack, Barry

Haacker, Erin

Haapanen, Reija

Haas, Rüdiger

Hackel, Timo

Hackert, Eric

Hadas, Tomasz

Hadjimitsis, Diofantos

Haest, Birgen

Hagenlocher, Michael

Haggerty, Julie

Haghighattalab, Atena

Hagolle, Olivier

Haidu, Ionel

Hailemariam, Sisay Nune

Hajder, Levente

Hajduch, Guillaume

Hakkenberg, Christopher

Hakuba, Maria

Haladová, Zuzana Berger

HALIK, Ümüt

Hall, Joanne

Halla, Jamie

Haller, Merrick

Halls, Joanne

Häme, Tuomas

Hamed, Ali

Hamunyela, Eliakim

Hamza, Ben

Han, Dawei

Han, Guoqi

Han, Hyangsun

Han, Jungong

Han, Junwei

Han, Soohee

Han, Xianhua

Han, Youkyung

Hancock, Craig Matthew

Hand, Jenny

Hanink, Dean

Hänsch, Ronny

Hanschmann, Timo 


\author{
Hao, Pengyu \\ Harb, Mostapha \\ Harder, Phillip \\ Hardiman, Brady \\ Harding, Richard \\ Hardy, Andy \\ Hare, Timothy \\ Harguess, Josh \\ Harris, Paul \\ Harwin, Steve \\ Hasager, Charlotte \\ Hashimoto, Manabu \\ Hassan, Quazi \\ Hatfield, Jerry \\ Haut, Juan Mario \\ Havenith, Hans-Balder \\ Havivi, Shiran \\ Hawlader, Muhammad Farhad \\ Hayasaka, Hiroshi \\ Hayashi, Masato \\ $\mathrm{He}$, Cenlin \\ $\mathrm{He}$, Chao \\ He, Fangning \\ $\mathrm{He}$, Jian \\ He, Liming \\ He, Luxiao \\ He, Mingzhu \\ He, Xianqiang \\ He, Xiongkui \\ He, Yaqian \\ He, Yuxiang \\ Healey, Sean \\ Hebel, Marcus \\ Heenkenda, Muditha \\ Heiden, Uta \\ Heikkonen, Jukka \\ Heinzel, Johannes \\ Heiselberg, Henning \\ Hejazin, Yazan \\ Hejmanowski, Ryszard \\ Helder, Dennis \\ Helm, Veit \\ Helman, David
}

Helmi, Ghanmi

Henderson, Mark

Henderson, Stephanie

Henebry, Geoffrey

Henriques, Renato Filipe

Henry, Mary

Heo, Moonbeom

Heras, Dora Blanco

Herbei, Mihai Valentin

Hermosilla, Txomin

Hernández, Armand

Hernández, David

Hernandez, Monica

Hernandez-Marín, Martín

Heron, Malcolm

Herrera, Lorena

Herrmann, Ittai

Hess, Laura

Hesse, Ralf

Hesselbarth, Anja

Hethcoat, Matthew

Heuzé, Céline

Hewson, Michael

Hewson, Rob

Hidalgo, Miguel Ángel Moreno

Hieronymi, Martin

Hilberg, Sylke

Hill, Joachim

Hill, Michael

Hill, Victoria

Hillger, Don

Hirata, Takafumi

Hirata, Yasumasa

Hird, Jennifer

Hirooka, Yoshihiro

Hirota, Nagio

Hirsch, Ernest

Hirschmugl, Manuela

Hisaki, Yukiharu

Ho Tong Minh, Din

Ho, Chung-Ru

Hobiger, Thomas

Hochberg, Eric 


\begin{tabular}{|c|c|}
\hline Hochschild, Volker & Huang, Haiyan \\
\hline Hocke, Klemens & Huang, Huei-Ping \\
\hline Hof, Anouschka & Huang, Jianxi \\
\hline Hoffmann, Lars & Huang, Mong-Han \\
\hline Hoffmeister, Dirk & Huang, Weimin \\
\hline Hofmann, Peter & Huang, Wenli \\
\hline Höger, Marek & Huang, Xiaodong \\
\hline Højstrup, Jørgen & Huang, Xin \\
\hline Hölbling, Daniel & Huang, Yan \\
\hline Hollaus, Markus & Huang, Yanbo \\
\hline Holley, Rachel & Huang, Yi \\
\hline Holman, Fenner & Huang, Yishuo \\
\hline Holt, Benjamin & Huang, Yu \\
\hline Holt, Tom & Hudak, Andrew \\
\hline Homayouni, Saeid & Huesca, Margarita \\
\hline Homolová, Lucie & Huettmann, Falk \\
\hline Hong, Chang-Ki & Huffman, George \\
\hline Hong, Hyunki & Hughes, Christopher \\
\hline Hong, Yulan & Hughes, M. Joseph \\
\hline Honkavaara, Eija & Humagain, Kamal \\
\hline Hoque, M Mainul & Humphrey, Vincent \\
\hline Hornbuckle, Brian & HUNG, HO-LUNG \\
\hline Horng, Shi-jinn & Hung, Ming-Chih \\
\hline Horváth, Ákos & Hunt, Earle R \\
\hline Hoshino, Buho & Huntemann, Marcus \\
\hline Hossain, Azad & Huo, Lian-Zhi \\
\hline Hossain, Mohammad Shawkat & Husak, Gregory \\
\hline Hostache, Renaud & Husson, Romain \\
\hline Houben, Sebastian & Huston, Dryver \\
\hline Houborg, Rasmus & Hwang, Dong-Hwan \\
\hline Houlié, Nicolas & Hwang, Wen-Liang \\
\hline Hovi, Aarne & Hwang, Yoola \\
\hline Hseu, Zeng-Yei & Hyakawa, Yuichi \\
\hline Hsu, Feng Chi & Iannelli, Gianni Cristian \\
\hline $\mathrm{Hu}$, Feng & Ibaraki, Yasuomi \\
\hline $\mathrm{Hu}$, Jry-Ching & Ibarrola, Edurne \\
\hline Hu, Weifei & Ibrahim Salem, Salem \\
\hline Huang, Chang & Ibrahim, Ali \\
\hline Huang, Changchun & Ichii, Kazuhito \\
\hline Huang, Chong & Ichikawa, Kaoru \\
\hline Huang, Guang-Bin & Ichinose, Toshiaki \\
\hline Huang, Guangwei & Ide, Reiko \\
\hline Huang, Guanyu & Ienco, Dino \\
\hline
\end{tabular}


Iervolino, Pasquale

Igoe, Damien

Iiames, John

Iizuka, Kotaro

Iliyasu, Abdullah

Im, Jungho

IM, Nam-Kyun

Imai, Nilton

Imasu, Ryoichi

Imbrenda, Vito

Immitzer, Markus

Imperatore, Pasquale

Inamdar, Anand

Inggs, Michael

Inglada, Jordi

Inoue, Yoshio

Intrieri, Emanuele

Ioannidis, Charalabos

Iodice, Antonio

Iordache, Marian-Daniel

Iqbal, Faheem

Irulappa Pillai Vijayakumar, Dinesh Babu

Irvine-Fynn, Tristram

Ishibe, Takeo

Ishida, Haruma

Ishidara, Hiroshi

Ishitsuka, Kazuya

Ishtiaque, Asif

Isioye, Olalekan

Israel, Martin

Itahashi, Syuichi

Ivanovici, Mihai

Ivashov, Sergey

Ivezic, Vedran

Iwasaki, Akira

Iwaszczuk, Dorota

Izquierdo Verdiguier, Emma

Izquierdo, Alberto

Izquierdo, Estibaliz Martinez

J. Caruso, Michael

J. G. Teunissen, Peter

Jabari, Shabnam

Jacobsen, Karsten
Jacobsen, Knut Stanley

Jaeggli, Barbara George

Jaferzadeh, Keyvan

Jahangiri Koohbanani, Behnam

Jäkel, Evelyn

Jakimow, Benjamin

Jakobsson, Antti

Jakovac, Catarina

Jakovels, Dainis

Jalal, Ahmad

Jalalvand, Azarakhsh

Jamali, Mohsin

Jamali, Sadegh

James, Mark

James, Mike

Jamet, Cedric

Jamil, Noreen

Jan, Chyan-long

Janicki, Grzegorz

Jankowski, Łukasz

Jansen, Robert

Jansing, David

Janus, Jaroslaw

Jarabo-Amores, María Pilar

Jaramillo, Fernando

Jat, Prahlad

Javankhoshdel, Sina

Jaw, Jen-Jer

Je, Changsoo

Jeansoulin, Robert

Jeng, Yih

Jensen, Jennifer

Jensen, Tim Enzlberger

Jeong, Seongsu

Jeong, Ukkyo

Ji, Kefeng

Ji, Yaqi

Jia, Mingming

Jiang, Jonathan

Jiang, Lingmei

Jiang, Wanshou

Jiao, Licheng

Jiménez, Enrique 


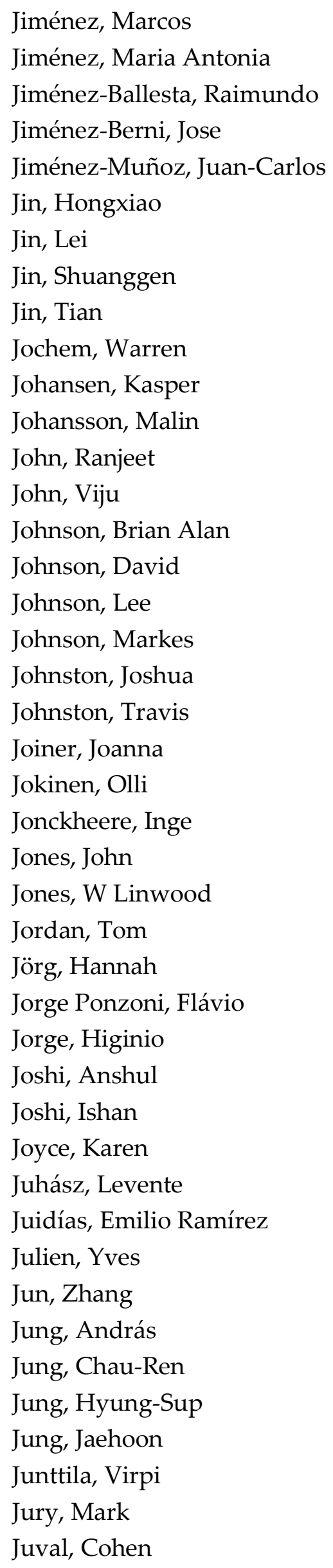

Kacev, Dovi

Kahn, Brain

Kaim, Dominik

Kaimaris, Dimitrios

Kalacska, Margaret

Kalayci, Tuna

Kallel, Abdelaziz

Kalogiros, John

Kalomiros, John

Kambezidis, Harry

Kamencay, Patrik

Kamilaris, Andreas

Kamini, Yadav

Kampel, Milton

Kanakoudis, Vasilis

Kanavos, Andreas

Kandasamy, Sivasathivel

Kandus, Patricia

Kane, Van

Kang, Li-Wei

Kang, Sung Ha

Kang, Xudong

Kang, Yanghui

Kang, Zhizhong

Kania, Adam

Kant, Surya

Kanuk, Jan

Kao, Hsun-Ying

Kaplan, Gordana

Kaplon, Jan

Kapuscinski, Tomasz

Karagiannidis, Athanasios

Karami, Mojtaba

Karantzalos, Konstantinos

Karimi, Hassan

Karimova, Svetlana

Karimzadeh, Sadra

Karine, Ayoub

Karjalainen, Mika

Karki, Ramchandra

Karthe, Daniel

Karvonen, Juha

Kasprzak, Marek 
Katagis, Thomas

Kateris, Dimitrios

Katsanos, Dimitris

Katsoulas, Nikolaos

Kattenborn, Gilbert

Katuwal, Sheela

Kauranne, Tuomo

Kavroudakis, Dimitris

Kawamura, Kensuke

Kazakis, Nerantzis

Kazanskiy, Nikolay

Kazmi, Jamil

Kaźmierowski, Cezary

Kearney, Michael

Kedzierski, Michal

Kefauver, Shawn

Kefauver, Shawn Carlisle

Keith, Darryl

Kekec, Taygun

Kelley, Lisa

Kelly, Maggi

Kenlo, Nasahara

Kennedy, Robert

Kent, Rafi

Kerekes, John

Kereszturi, Gabor

Kermarrec, Gael

Kern, Aniko

Kern, Christoph

Kern, Stefan

Kersten, Tobias

Keskinarkaus, Anja

Kettunen, Pyry

Khalaf, Walaa

Khaloo, Ali

Khamukhin, Alexander

Khankhoje, Uday

Khanna, Raghav

Khanna, Shruti

Khatami, Reza

Khatri, Pradeep

Khazaei, Bahram

Kheireddine, Malika
Khenchaf, Ali

Kheyrollah Pour, Homa

Khodabandeh, Amir

Khoshelham, Kourosh

Khosrawi, Farahnaz

Khwaja, Ahmed Shaharyar

Kia, Seyed Mostafa

Kiang, Jean-Fu

Kiefer, Johannes

Kiemle, Christoph

Kikuchi, Nobuhiro

Kim, Choonsig

Kim, Dohyeong

Kim, Dukhyeon

Kim, EunHee

Kim, Hae-Cheol

Kim, Hakjin

Kim, Hyun-cheol

Kim, Jeongrae

Kim, Jin-Woo

Kim, Jung-Rack

KIM, KyoungSik

Kim, Seongjoon

Kim, Seungbum

Kim, Sungho

Kim, Sungwon

Kim, Taejung

Kim, Tae-Sung

Kim, Youngwook

Kimoto, Shigeru

Kindu, Mengistie

King, Diana

King, Douglas

Kinnard, Christophe

Kinoshita, Tsuguki

Kira, Zsolt

Király, Géza

Kirkko-Jaakkola, Martti

Klamerus-Iwan, Anna

Klauberg, Carine

Kleftogiannis, Dimitrios

Klem, Karel

Klos, Anna 
Kneen, Melanie Anne

Kniffka, Anke

Knoth, Christian

Knudby, Anders

Ko, Byoung Chul

Ko, Connie

Ko, Kwang Hee

Kobayashi, Hideki

Kobayashi, Hirokazu

Koch, Julian

Koch, Magaly

Koci, Jack

Kociuba, Waldemar

Kodet, Jan

Kodors, Sergejs

Koedsin, Werapong

Koeva, Mila

Koh, Jaehan

Köhl, Michael

Kokkalis, Panagiotis

Kolassa, Jana

Kolecka, Natalia

Kolerski, Tomasz

Kolios, Stavros

Kolokoussis, Pol

Komarov, Alexander

komatsu, teruhisa

Konstantakopoulou, Foteini

Kontoes, Haris

Konushin, Anton

Kooistra, Lammert

Kool, Dilia

Kopackova, Veronika

Kopecká, Monika

Kopelevich, Oleg

Koračin, Darko

Kordi Ghasrodashti, Elham

Koreň, Milan

Korets, Mikhail

Korkin, Sergey

Korrapati, Hemanth

Korsgaard, Niels

Kosmopoulos, Panagiotis
Kostamovaara, Juha

Kostelecký, Jakub

Kotarba, Andrzej

Kothe, Steffen

Kotthaus, Simone

Kouadio, Louis

Kouba, Jan

Koukoulas, Sotirios

Koukouvelas, Ioannis

Kourgialas, Nektarios

Kourkouli, Penelope

Koutsias, Nikolaos

Koutsias, Nikos

Koutsoudis, Anestis

Kouyama, Toru

Kouziokas, Georgios

Kowalczyk, Kamil

Kozhoridze, Giorgi

Kpalma, Kidiyo

Kraatz, Simon

Krakauer, Nir

Kramer, Louisa

Kraszewski, Bartłomiej

Krawczyk, Bartosz

Krayenhoff, Scott

Kregar, Klemen

Krishna Gumma, Murali

Krishna Moorthy, Sruthi

Krishnamurthy, Raghavendra

Kritikos, Kyriakos

Kroczak, Rafal

Kropáček, Jan

Kryjak, Tomasz

Krzystek, Peter

Ku, Nian-Wei

Kudela, Raphael

Kudo, Rei

Kudryavtsev, Vladimir

Kuemmerle, Tobias

Kuenzer, Claudia

Kuffer, Monika

Kuhlmann, Gerrit

Kühn, Martin 
Kulawiak, Marcin

Kumar, Kanike Raghavendra

Kumar, Uttam

Kumar, Vinay

Kummerow, Christian

Kuntz, Steffen

Kuo, Chaincy

Kupkova, Lucie

Kurekin, Andriy

Kuremoto, Takashi

Kurihara, Yukio

Kurkina, Oxana

Kurz, Franz

Kusnierek, Krzysztof

Kussul, Nataliia

Kutnjak, Hrvoje

Kutser, Tiit

Kuželka, Karel

Kwak, Youngjoo

Kwan, Chiman

Kwok, Ngai Ming

Kwon, Ohseok

Kycko, Marlena

Kyriakides, Nicholas

La, Tran Vu

Łabędzki, Leszek

Lacava, Teodosio

Lacaze, Bernard

Laefer, Debra

Lagouarde, Jean-Pierre

Lagüela López, Susana

Lahoz, William

Laiolo, Marco

Lakshmi, Venkat

Lamare, Maxim

Lambrou, Tryphon

Lamparelli, Rubens Augusto Camargo

Lanaras, Charis

Lanari, Riccardo

Land, Peter

Landmann, Tobias

Landrieua, Loïc

Landulfo, Eduardo
Lane, Charles

Lane-Serff, Gregory

Laneve, Giovanni

Lanfredi, Maria

Langford, Zachary

Langhammer, Jakub

Längkvist, Martin

Langland, Rolf

Lannoy, Gabrielle De

Lanorte, Antonio

Lapucci, Chiara

Lari, Zahra

Larocca, Ana Paula

LaRue, Elizabeth

LaRue, Michelle

Lasko, Kristofer

Lasky, Ty

László, Czúni

Lathrop, Richard

Latifi, Hooman

Latifovic, Rasim

Lau, Lawrence

Laukamp, Carsten

Launeau, Patrick

Lavalle, Marco

Lavine, Barry

Lavoué, François

Lavrova, Olga

Lawniczak-Malińska, Agnieszka

Lawrence, Dan

Laws, Edward A.

Lázaro, Clara

Lázaro, José L.

Lazarov, Andon

Lazecky, Milan

Le Bas, Tim

Le Bris, Arnaud

Le Louarn, Marine

Le Maire, Guerric

Le Moan, Steven

Le Pennec, Erwan

Leblanc, George

Leccese, Fabio 
Lecours, Vincent

Lee, Bora

Lee, Byun-Woo

Lee, Eunmok

Lee, Hai-Tien

Lee, Hyongki

Lee, Hyungtae

Lee, Ju

Lee, Jun-Hak

Lee, Kiwon

Lee, Moonjin

Lee, Sang-Il

Lee, Saro

Lee, Suk Jin

Lee, Temple

Lee, Wei-Liang

Lee, Woo-Kyung

Lee, Yang-Won

Lee, Zhongping

Leelőssy, Ádám

Leeuw, Gerrit de

Lefevre, Sebastien

Legaard, Kasey

Legleiter, Carl

Lehmann, Eric

Lehmann, Moritz

Lehnert, Lukas

Lehr, Bill

Lehtola, Ville

Leifer, Ira

Leinonen, Jussi

Lemmens, Mathias

Lemoine, Guido

Leng, Pei

Lennert, Moritz

Lenz-Wiedemann, Victoria

Léon, Jean-francois

Leonowicz, Zbigniew

Lepage, Richard

Lerga, Jonatan

Lerner, Amit

Lerot, Christophe

Leroux, Louise
Lesiv, Myroslava

Leucci, Giovanni

Leung, Hui Min

Levashova, Natalia

Leverington, David

Levick, Shaun

Levin, Gregor

Levine, Judah

Lewis, Jasper

Lewis, Quinn W.

Lezzerini, Marco

Lhermitte, Stef

Li, Dengqiu

Li, Deren

Li, Guiying

Li, Heng-Chao

Li, Jingjing

Li, Kezhi

Li, Liwei

Li, Long

Li, Peijun

Li, Rui

Li, Siwei

$\mathrm{Li}$, Tao

Li, Weidong

Li, Wenjuan

Li, Wenliang

$\mathrm{Li}, \mathrm{Xi}_{\mathrm{i}}$

Li, Xin

Li, Xueke

Li, Yanan

Li, Yansheng

Li, Yue

Li, Yunhua

Li, Zhan

Li, Zhaoguo

Li, Zhaoliang

Li, Zhenhai

Li, Zhongbin

Li, Zishen

Li, Zuchuan

Liang, Binqing

Liang, X.M. 
Liang, Xuefeng

Liao, Mingsheng

Liao, Tien-Hao

Libohova, Zamir

Libonati, Renata

Licer, Matjaz

Lichti, Derek

Liebisch, Frank

Liesenberg, Veraldo

Lieser, Jan

Liknes, Greg C.

Lilienthal, Holger

Lillo-Saavedra, Mario

Lim, Deok Won

Lim, Samsung

Lin, Aiwen

Lin, Chien-Chou

Lin, Chingweei

Lin, Chinsu

Lin, Feng-Cheng

Lin, Jianmin

Lin, Jianzhe

Lin, Liao-Fan

Lin, Wenming

Lin, Yang

Lin, Yun

Lindberg, Eva

Lininger, Katherine

Lipa, Belinda

Lipping, Tarmo

Lipponen, Antti

Lira, Cristina

Lisi, Mariano

Litvinov, Pavel

Liu, Chao

Liu, Chunwei

Liu, Datong

Liu, Dong

Liu, Guigen

Liu, Hongyan

Liu, Kai

Liu, Keng-Hao

Liu, Lin
Liu, Lingling

Liu, Ning

Liu, Pang-Wei

Liu, Peng

Liu, Qi

Liu, Ronghua

Liu, Shufan

Liu, Sicong

Liu, Suxing

Liu, Tao

Liu, Wei-Min

Liu, Xianglin

Liu, Xiaoming

Liu, Xiaoyang

Liu, Yan

Liu, Yonggang

Liu, Yuling

Liu, Yuming

Liu, Zhen

Liu, Zhong

Livens, Stefan

Livsey, Daniel

Lo Brutto, Mauro

Lofgren, Brent

Logan, Timothy

Lohr, Dieter

Lolli, Simone

Long, Nathalie

Longo, Sandro

Loomis, Bryant

Lopatin, Javier

Lopez-Baeza, Ernesto

Lopez-Dekker, Paco

Lopez-Granados, Francisca

López-Serrano, Pablito

Lorenzo Trueba, Jorge

Los, Sietse

Lottes, Philipp

Lou, Der-Chyuan

Loures, Luis

Lovas, Tamás

Lowe, Kelsey

Lu, Ching-Ta 
Lu, Dengsheng

Lu, Hui

Lu, Linlin

Lubczonek, Jacek

Lucieer, Arko

Ludeno, Giovanni

Luhmann, Thomas

Lui, Antonny

Lukač, Niko

Lukeš, Petr

Lukin, Vladimir

Luks, Bartłomiej

Lund, Björn

Lundin, Ola

Luo, Tao

Luo, Yi

Luther, Joan

Luzi, Guido

Lyapustin, Alexei I

Lymburner, Leo

Lyons, Evan

Lyzenga, David

M. M. Kahaki, Seyed

M.H.Baker, David

Ma, Dandan

Ma, Jiayi

Ma, Lei

Ma, Ting

Ma, Weiqiang

$\mathrm{Ma}, \mathrm{Wu}$

Maas, Hans-Gert

Maboudi, Mehdi

Macher, Hélène

Machida, Toshinobu

Macinnis-Ng, Cate

Mackenzie, Jock

Madani, Nima

Madhavan, Sriharsha

Madurapperuma, Buddhika

Maffei, Carmine

Magarey, Roger

Magdon, Paul

Maggioni, Viviana
Maggiori, Emmanuel

Magliulo, Vincenzo

Magnani, Federico

Magney, Troy

Magnussen, Steen

Magnusson, Martin

Magri, Fabien

Magruder, Lori

Mahabir, Ron

Mahanama, Sarith

Mahapatra, Chinmaya

Mahmoud, Mahmoud Ibrahim

Mahmud, Rizaludin

Mahoney, Robert

Maiello, Ida

Maignan, Fabienne

Maimaitiyiming, Matthew

Maingi, John

Majurec, Ninoslav

Makantasis, Konstantinos

Makris, Nicholas

Mäkynen, Marko

Malakar, Nabin

Malambo, Lonesome

Malbeteau, Yoann

Malek, Žiga

Malinverni, Eva Savina

Mallet, Marc

Mallick, Javed

Mallinis, Giorgios

Mallorqui, Jordi

Malone, Brendan

Maltese, Antonino

Manconi, Andrea

Mandanici, Emanuele

Mandlburger, Gottfried

Manfreda, Salvatore

Mangiarotti, Sylvain

Manickam, Surendar

Mansberger, Reinfried

Mansi, Ahmed Hamdi

Manso Callejo, Miguel Ángel

Mantas, Vasco 
Mantovani, Matteo

Manzo, Ciro

Mao, Dehua

Marapareddy, Ramakalavathi

Marcello, Javier

Marcolin, Federica

Marconi, Sergio

Marcotegui, Beatriz

Marengo, Jose

Maresma, Ángel

Mărgărint, Mihai Ciprian

Marghany, Maged

Mariani, Stefano

Marin, Andrea

Marinello, Francesco

Mariotto, Isabella

Marjanović, Hrvoje

Mark, Ole

Markelin, Lauri

Markert, Kel

Markham, Brian

Markos, Nikos

Marmorino, George

Marpu, Prashanth Reddy

Marques, Fernando

Marseille, Gert-Jan

Marsh, Stuart

Marshall, Michael

Martens, Brecht

Martí, Pau

Martin, Tara

Martín-Crespo, Tomás

Martinez, Beatriz

Martinez, Joaquin

Martínez, Justino

Martinez, Patricio

Martínez-Álvarez, Francisco

Martínez-De-Dios, José Ramiro

Martinez-Espejo Zaragoza, Isabel

Martínez-Fernández, José

Martínez-García, Edgar

Martínez-Graña, Antonio

Martinez-Izquierdo, Estibaliz
Martinez-Tossas, Luis Antonio

Martins, João

Martinuzzi, Sebastian

Martone, Michele

Marullo, Salvatore

Marx, Andrew

Mas, Jean-Francois

Mascolo, Lucio

Maselli, Fabio

Masiello, Guido

Masiero, Andrea

Masih, Ilyas

Masini, Nicola

Mason, Philippa

Masoumi, Majid

Maspataud, Aurélie

Massari, Christian

Masse, Antoine

Masud, M Badrul

Matano, Fabio

Mateo-Lázaro, Jesús

Matese, Alessandro

Mateus, Pedro

Matheou, Georgios

Matsoukas, Christos

Matsunaga, Tsuneo

Matsuoka, Masayuki

Matsushima, Dai

Mattar, Cristian

Matteoli, Stefania

Matthews, Grant

Matthews, Jessica

Mattia, Francesco

Maturi, Eileen

Mauro, Francisco

Mauro, Giovanni

Maus, Victor

Mauser, Wolfram

Maxwell, Aaron

Maze, Guillaume

Mazher, Abeer

Mazurek, Przemysław

Mazziotta, Adriano 


\author{
Mazzola, Mauro \\ Mazzoleni, Maurizio \\ McCall, Michael \\ McCarty, Jessica \\ McFall, Kevin \\ McGaughey, Robert \\ McGuire, Patrick \\ McKerrow, Alexa \\ McNabb, Robert \\ McNeil, Brenden \\ Mcneill, Stephen \\ McRoberts, Ronald \\ Meersmans, Jeroen \\ Meggio, Franco \\ Mehan, Sushant \\ Mei, Linlu \\ Mei, Shao-Hui \\ Mei, Yiwen \\ Meier, Walter \\ Meisina, Claudia \\ Meissner, Thomas \\ Melendez-Pastor, Ignacio \\ Meleti, Charikleia \\ Melis, Maria Teresa \\ Melnichenko, Oleg \\ Melnikov, Valery \\ Melo Damian, Júnior \\ Melo-Pinto, Pedro \\ Melvin, William \\ Mencin, David \\ Mendes, Renato \\ Mendes, Virgilio \\ Méndez Fuentes, Valeriano \\ Meneghini, Robert \\ Menezes, Viviane \\ Meng, Ran \\ Menna, Fabio \\ Menzies, Robert \\ Mercado, Jesús \\ Mercado-Carmona, Jesús \\ Merciol, François \\ Merino, Silvia \\ Merlaud, Alexis
}

Merlin, Olivier

Mermoz, Stéphane

Meron, Moshe

Merrelli, Aronne

Merry, Krista

Merryman Boncori, John Peter

Mesas Carrascosa, Francisco Javier

Meschino, Simone

Messias Pereira Nogueira, Joana

Meyer, Victoria

Mia, Md. Bodruddoza

Mialon, Amaud

Michał, Kędzierski

Michalski, Adam

Michelini, Alberto

Michez, Adrien

Michieletto, Stefano

Micijevic, Esad

Midgley, Nicholas

Mielke, Christian

Miernecki, Maciej

Mietzner, Jan

Migoya-Orue, Yenca

Mihai, Laura

Mikhailova, Elena

Mikita, Tomáš

Milan, David

Miles, Evan

Milesi, Cristina

Milewski, Adam

Milillo, Pietro

Militino, Ana Fernández

Millan Almaraz, Jesus Roberto

Millán, Luis

Millard, Koreen

Miller, Boris

Miller, Steven

Mills, Steven

Min, Jinzhong

Mineo, Simone

Minghini, Marco

Minor, Jesse

Mira, Maria 
Miralles, Diego

Miranda, Pedro

Mishra, Bhogendra

Mishra, Kumar Vijay

Mishra, Sachidananda

Mishra, Vikalp

Misra, Anupam

Misra, Gourav

Mitchell, Catherine

Mitraka, Zina

Miura, Hiroyuki

Miyazaki, Hiroyuki

Mizuochi, Hiroki

Młyński, Dariusz

$\mathrm{Mo}, \mathrm{Yu}$

Mobasheri, Amin

Mobley, Curtis

Möckel, Thomas

Modala, Naga Raghuveer

Modica, Giuseppe

Moghadas, Davood

Mogo, Sandra

Mohamed, Yasir

Mohammadzadeh, Ali

Mohan, Midhun

Mohd. Shafri, Helmi Zulhaidi

Mojaddadi Rizeei, Hossein

Moknatian, Mahrokh

Mokros, Martin

Molina, Iñigo

Molina, Jose Luis

Molina, Pere

Molinier, Matthieu

Möller, Markus

Møller-Jensen, Lasse

Molloy, Timothy

Momm, Henrique

Mona, Lucia

Monaldo, Frank

Monasse, Pascal

Mondal, Arun

Mondal, Pinki

Mondal, Surabuddin
Mondini, Alessandro

Mongil-Manso, Jorge

Mongus, Domen

Monico, Joao Francisco Galera

Monserrat, Oriol

Monsivais-Huertero, Alejandro

Montagnani, Leonardo

Monteiro Gonçalves, José Manuel

Monterroso-Checa, Antonio

Montes, Marcos

Monti, Paolo

Montoro, Amelia

Montpetit, Benoit

Montrucchio, Bartolomeo

Montuori, Antonio

Moon, Hyeonjoon

Moore, Nathan

Moore, Timothy

Mora, Andre

Mora, Carla

Morales Esteban, Antonio

Moreno, Adam

Moreno, Jose

Moreno-Madrinan, Max

Moretti, Sandro

Morey, Steve

Morey, Steven

Mori, Saverio

Morini, Elena

Moritsuka, Naoki

Moriyama, Toshifumi

Moroni, Davide

Morris, Brendan

Morris, Edward

Morris, Tim

Morrison, Keith

Morsy, Salem

Mortlock, Thomas

Moser, Gabriele

Moshou, Dimitrios

Mota, Bernardo

Motagh, Mahdi

Mott, Rowan 
Motte, Erwan

Mõttus, Matti

Mou, Lichao

Mouillot, Florent

Mousivand, Ali

Moustakidis, Serafeim

Mozos, Daniel

Mozzi, Paolo

Mroz, Marek

$\mathrm{Mu}$, Mingquan

$\mathrm{Mu}$, Xihan

Mubea, Kenneth

Mueller, Rupert

Mui, Amy

Mukai, Sonoyo

Mukherjee, Kriti

Mulas, Marco

Mulianga, Betty

Müller Schmied, Hannes

Müller, Markus Uwe

Müller, Richard

Mullerova, Jana

Mullissa, Adugna

Münch, Zahn

Mund, Jan-Peter

Munir, Tariq

Muntendam-Bos, Annemarie

Murböck, Michael

Murgante, Beniamino

Murillo, Ana Cristina

Muro, Javier

Murphy, James

Murphy, Maurice

Muschinski, Andreas

Mushini, Sajan

Muskett, Reginald

Mutiibwa, Denis

Muukkonen, Petteri

Mylona, Eleftheria

$\mathrm{Na}$, Wongi

Naeger, Aaron

Naeije, Marc

Næsset, Erik
Nagai, Hiroto

Nagaike, Takuo

Nahid, Abdullah-Al

Naik, Ganesh

Naito, Adam

Najibi, Nasser

Nakaji, Tatsuro

Nalepa, Jakub

Nam, SungHyun

Nanni, Loris

Napiórkowska-Krzebietke, Agnieszka

Nardi, Fernando

Nardino, Vanni

Narouei-Khandan, Hossein

Nascetti, Andrea

Natsuaki, Ryo

Naud, Catherine

Navarro Ferreira, Ana

Navarro Pedreño, Jose

Navarro, Gabriel

Nawaf, Mohamad Motasem

Nayar, Anand

Nazeer, Majid

Nebiker, Stephan

Negri, Rogério Galante

Neigh, Christopher

Nekrasov, Alexey

Nelson, Andy

Neri, Paolo

Nerry, Françoise

Neteler, Markus

Netzband, Maik

Neumann, Mathias

Nevalainen, Olli

Nevalainen, Paavo

Newlands, Nathaniel

Newsam, Shawn

Newton, Andrew

$\mathrm{Ng}$, Alex Hay-Man

$\mathrm{Ng}$, Wai-Tim

Ngoy, Kikombo

Nguyen, Duy Ba

Nguyen, Lan 
Nguyen, Uyen

Nhamo, Luxon

Niccolai, Alessandro

Nico, Giovanni

Nicodemo, Gianfranco

Nicol, John

$\mathrm{Nicu}$, Ionut Cristi

Niculita, Mihai

Nie, Sheng

Nielsen, Karina

Nikolakopoulos, Konstantinos

Nikolopoulos, Efthymios

Nilfouroushan, Faramarz

Nilo, Saverio Teodosio

Nilsson, Tobias

Ningthoujam, Ramesh

Nino, Pasquale

Ninyerola, Miquel

Nirchio, Francesco

Niro, Fabrizio

Niroumand-Jadidi, Milad

Nishizawa, Tomoaki

Nissi, Eugenia

Nistor, Sorin

Niyonsenga, Theo

Nkwunonwo, Ugonna

Nobile, Adriano

Nobrega, Rodolfo

Nocelo Lopez, Ruben

Nocerino, Erica

Noda, Hibiki

Noël, Stefan

Nogueira, Miguel

Noh, Seong Jin

Noh, Youngmin

Noi, Phan Thanh

Nolan, Rachael

Nolè, Gabriele

Noszczyk, Tomasz

Nothdurft, Arne

Notti, Davide

Novack, Tessio

Novak, Pavel
Novelli, Antonio

Novellino, Alessandro

Noviello, Carlo

Novillo, Carlos

Novoa, Stéfani

Nowacki, Greg

Nuimura, Takayuki

Nuñez Andrés, Amparo

Nurzynska, Karolina

Nüsser, Marcus

Nykiel, Grzegorz

Nyman, Petter

O'Connor, Brian

Obata, Kenta

O'Connell, Mark

Oeser, Julian

Ogashawara, Igor

Ogen, Yaron

Ogilvie, Andrew

Ogneva-Himmelberger, Yelena

Oguchi, Takashi

Ogundipe, Oluropo

Oh, Sangyoon

Oh, Yisok

Ohashi, Hiroki

Okarma, Krzysztof

Okuda, Masahiro

Okujeni, Akpona

Okuyama, Arata

Okyay, Unal

Okyay, Ünal

Olander, Timothy

Oldeland, Jens

Oliphant, Adam

Oliveira, Henrique

Olmedo, Estrella

Olofsson, Kenneth

Olofsson, Pontus

O'Loughlin, Fiachra

Olpenda, Alex

Olsen, Michael

Olsson, Håkan

Olsson, PerOla 
Olthof, Ian

Omranian, Ehsan

Ondicol, Ramón Pellitero

Oneto, Luca

Oniga, Florin

Oniga, Valeria-Ersilia

Oommen, Thomas

Opitz, Rachel

Opitz, Rachel S.

Opromolla, Roberto

Ordoñez, Celestino

Orfila, Alejandro

Organelli, Emanuele

Orlandini, Stefano

Ortega Huerta, Miguel

Ortega, Samuel

Osborn, Jon

Osinska-Skotak, Katarzyna

Osman, Mohammed

Osuch, Tomasz

Oszust, Mariusz

Ota, Tetsuji

Otčenášová, Alena

Otis, Daniel

Otremba, Zbigniew

Otsuka, Yuichi

Ottlé, Catherine

Ouchi, Kazuo

Oude Elberink, Sander

Ouled Sghaier, Moslem

Ouyang, Xiaoying

Ouzounov, Dimitar

Ozdemir, Ibrahim

Ozer, Ekin

Pabi, Opoku

Pablos, Miriam

Pacheco-Labrador, Javier

Padmanaban, Rajchandar

Paduan, Jeff

Pagliari, Diana

Paheding, Sidike

Pajewski, Lara

Pal, Mahesh
Pal, Sandip

Palace, Michael

Palamartchouk, Kirill

Palazzi, Elisa

Palazzo, Francesco

Pallotta, Luca

Palme, Massimo

Palmerini, Giovanni

Paloscia, Simonetta

Palubinskas, Gintautas

Pampaloni, Paolo

Pan, Haozhi

Pan, Zhigang

Panagiotakis, Costas

Panagiotidis, Dimitrios

Panagopoulos, Thomas

Panagos, Panos

Panda, Santosh

Panday, Dinesh

Pandey, Gunjan

Pandey, Prem

Panegrossi, Giulia

Panferov, Oleg

Pangaluru, Kishore

Pantazi, Xanthoula Eirini

Papa, Ivica

Papadopoulos, Nikos

Papakonstantinou, Apostolos

Papalou, Angeliki

Papanikolaou, Ioannis

Papanikolaou, Thomas

Papayannis, Alexandros

Papoutsis, Ioannis

Pappas, Odysseas A.

Parajka, Juraj

Parastatidis, David

Parcharidis, Issaak

Pardini, Matteo

Pardo, Miguel

Paredes, Franklin

Parente, Claudio

Parente, Leandro

Parida, Bikash 


\begin{tabular}{|c|}
\hline Paris, Claudia \\
\hline Park, Byungwoon \\
\hline Park, Chang-Hwan \\
\hline Park, Edward \\
\hline Park, Hogeun \\
\hline Park, Jihye \\
\hline Park, Kwandong \\
\hline Park, Kyungwon \\
\hline Park, No-Wook \\
\hline Park, Sang-Eun \\
\hline Park, Taejin \\
\hline Park, Yongeun \\
\hline Park, Youn Shik \\
\hline Parmehr, Ebadat G. \\
\hline Parmehr, Ebadat Ghanbari \\
\hline Parmentier, Frans-Jan \\
\hline Parmes, Eija \\
\hline Parmiggiani, Flavio \\
\hline Parrington, Mark \\
\hline Parrish, Christopher \\
\hline Pascual, Adrián \\
\hline Pascual, Josep E. Pardo \\
\hline Pascucci, Simone \\
\hline Pasian, Marco \\
\hline Pasolli, Edoardo \\
\hline Pasquali, Paolo \\
\hline Pasquariello, Guido \\
\hline Pasquero, Claudia \\
\hline Passaro, Marcello \\
\hline Pasternak, Mateusz \\
\hline Pastucha, Elżbieta \\
\hline Pata, Petr \\
\hline Patel, Nirav Nikunj \\
\hline Patias, Petros \\
\hline Patidar, Sandhya \\
\hline Pătrașcu, Carmen \\
\hline Paudel, Uttam \\
\hline Paugam, Ronan \\
\hline Paul, George \\
\hline Paule Bonnet, Marie \\
\hline Pauly, Klaas \\
\hline Pause, Marion \\
\hline Pavelka, Karel \\
\hline
\end{tabular}

Paweł, Pławiak

Pawlak, Michał

Payá, Prof. Dr. Luis

Payan, Christophe

Paynter, Ian

Paz, Igor

Paz-González, Antonio

Paziewski, Jacek

Pazur, Robert

Pearlmutter, David

Pedersen, Leif Toudal

Pegau, Scott

Pellarin, Thierry

Pelletier, Charlotte

Pellicani, Roberta

Pellitero, Ramon

Pellitero, Ramón

Pelto, Mauri

Peltoniemi, Jouni

Peng, Bin

Peng, Dongju

Peng, Ge

Peng, Jian

Peng, Jingjing

Peng, Xi

Peng, Zhengyu

Penner, Margaret

Pensieri, Sara

Penta, Bradley

Penttilä, Antti

Peón, Juanjo

Pepe, Antonio

Pepe, Massimiliano

Pepe, Susi

Peralta, Daniel

Pereira, Luísa

Perera, Kithsiri

Peres, Emanuel

Pérez González, María Eugenia

Pérez-Meana, Héctor Manuel

Perez-Priego, Oscar

Perez-Ramirez, Daniel

Pergola, Nicola 
Pergola, RNicola

Pering, Tom

Perko, Roland

Perroy, Ryan L.

Persello, Claudio

Persia, Fabio

Persson, Henrik Jan

Pesquer Mayos, Lluís

Petcu, Dana

Peter, Heike

Peter, Hofmann

Peters-Lidard, Christa D

Peterson, Birgit

Peterson, David

Peterson, Michael

Peterson, Peter

Peterson, Seth

Petillot, Yvan

Petit, Nicolas

Petkov, Boyan

Petlicki, Michal

Pętlicki, Michał

Petrakis, Roy

Petrellis, Nikos

Petropoulos, George

Petroselli, Andrea

Petsa, Elli

Pettinato, Simone

Pezzuolo, Andrea

Pfeffer, Julia

Pfeifroth, Uwe

Pfeil, Isabella

Pflanz, Michael

Pham, Minh-Tan

Pham, Tien Dat

Phang, Swee King

Piasecki, Michael

Picchio, Rodolfo

Pickell, Paul

Picone, Daniele

Picotte, Joshua

Pięciak, Tomasz

Pieraccini, Massimiliano
Pierce Jr, Kenneth B.

Piermattei, Livia

Pieters, Jan

Pimple, Uday

Pina, Pedro

Pindado, Santiago

Pindozzi, Stefania

Pinedo-Alvarez, Alfredo

Pingel, Thomas

Pinhasi, Yosef

Pinho, Carolina Moutinho Duque De

Pinho, Marcelo

Pinto, Francisco

Pinto, Naiara

Pinzón, Jorge

Piroddi, Luca

Piskozub, Jacek

Pissourios, Ioannis

Pitarch, Jaime

Pitkänen, Timo

Pizzolante, Raffaele

Plank, Simon

Pleskachevsky, Andrey

Plusquellic, David

Plyer, Aurélien

Poças, Isabel

Podobnikar, Tomaž

Poggi, Giovanni

Poggi, Prof. Giovanni

Poggio, Laura

Pohjola, Veijo

Polcari, Marco

Poli, Daniela

Poli, Paul

Policelli, Fritz

Polishchuk, Yury M.

Pollino, Maurizio

Polo-Gómez, María José

Pölönen, Ilkka

Polyakov, Alexander

Polykretis, Christos

Pomares, Luis Martin

Poméon, Thomas 
Pompilio, Loredana

Ponce, Octavio

Ponomarev, Evgenii

Pons, Xavier

Ponsford, Anthony

Pontius, Robert

Ponzoni, Flávio Jorge

Poortinga, Ate

Poostchi, Mahdieh

Popescu, Anca-Andreea

Popovic-Bugarin, Vesna

Popowicz, Adam

Porcar-Castell, Albert

Porcu, Federico

Porcù, Federico

Portillo, Carlos

Postylyakov, Oleg

Potter, Henry

Potter, Lee

Potterf, Maria

Pottier, Eric

Pottier, Julien

Poulain, Pierre-Marie

Pouliot, Darren

Pouliot, George

Pound, Michael

Pour, AB

Poursanidis, Dimitris

Poux, Florent

Povey, Adam

Prakash, Satya

Prasad, Abhnil

Prasad, Dilip

Praskievicz, Sarah

Prats-Iraola, Pau

Prechtel, Nikolas

Preissler, Jana

Presotto, Andrea

Prestininzi, Pietro

Prezioso, Giuseppina

Price, James

Price, Owen

Pricope, Narcisa
Prieto, Juan

Prochaska, Charikleia

Procter, Jonathan

Proctor, Cameron

Proud, Simon

Prudnikova, Elena

Przyborski, Marek

Psimoulis, Panos

Psomiadis, Emmanouil

Psuj, Grzegorz

$\mathrm{Pu}, \mathrm{R}$.

Pudelko, Rafal

Pugi, Luca

Pugliese Carratelli, Eugenio

Puletti, Nicola

Pulighe, Giuseppe

Puliti, Stefano

Pullanagari, Rajasheker

Purca, Sara

Purdy, Adam

Puthal, Deepak

Pyka, Krystian

Pytharoulis, Ioannis

Qamar, Faisal Mueen

Qi, Guanqiu

Qi, Wang

Qi, Yi

Qi, Zhixin

Qiao, Zhijun

Qihuan, Huang

Qin, Yi

Qiu, Jianxiu

Qiu, Tong

Qu, Ying

Quan, Xingwen

Queisser, Manuel

Rączkowski, Włodzimierz

Radoux, Julien

Radu-Daniel, Pintilii

Raffa, Francesco

Rahman, Hasibur

Rahman, Md. Shahinoor

Rahman, Mir Mustafizur 
Rahman, Moshiur

Rahman, Muhammad

Rahman, Muhammad Tauhidur

Rahman, MuhibUr

Rahnama, Mehdi

Rahnemoonfar, Maryam

Raja Abdullah, Raja Syamsul Azmir

Rajasekaran, Eswar

Rajib, Adnan

Rallo, Giovanni

Ramapriyan, Hampapuram

Ramella, Giuliana

Ramesh, Singh

Ramo, Rubén

Ramoelo, Abel

Rana, Parvez

Ranagalage, Manjula

Rangelova, Elena

Raouf, Abdul

Raoult, Vincent

Räsänen, Aleksi

Rasi, Rastislav

Raspini, Federico

Rasti, Behnood

Rasti, Pejman

Rasul, Azad

Ratto, Christopher

Räty, Minna

Raun, William

Raupach, Timothy Hugh

Rauste, Yrjo

Rautiainen, Kimmo

Rautiainen, Miina

Ravankar, Abhijeet

Ray, Ram

Raynolds, Martha

Razevig, Vladimir

Razionale, Armando

Re, Rebecca

Reagan, James

Reale, Diego

Reale, Ferdinando

Reath, Kevin
Reboul, Serge

Recanatesi, Fabio

Recondo, Carmen

Reddy Marpu, Prashanth

Redweik, Paula

Rees, Gareth

Reese, Heather

Refice, Alberto

Reggiannini, Marco

Regnard, Jean-Luc

Regnauld, Hervé

Reguzzoni, Mirko

Rehush, Nataliia

Reimann, Jens

Reinartz, Peter

Reinoso, Juan Francisco

Reinoso, Oscar

Reis, Mariane

Reitz, Meredith

Rejas Ayuga, Juan Gregorio

Remenyi, Tomas

Remmel, Tarmo

Remy, Dominique

Ren, Huazhong

Ren, Li

Ren, Lin

Renard, Jean-Baptiste

Rengarajan, Rajagopalan

Renné, David

Rennie, Susan

Renza, Diego

Repasky, Kevin

Requena-Mullor, Juan

Retscher, Günther

Reyes, Melissa

Rhea, Lee K.

Rhudy, Matthew

Riaza, Asuncion

Ribeiro, Eraldo

Ribeiro, Rita Cláudia Cardoso

Ribó, Serni

Ribolini, Adriano

Ricciardelli, Elisabetta 
Richard, Biancale

Richards, Daniel

Richardson, Tristam

Richards-Rissetto, Heather

Richter, Rudolf

Ricko, Martina

Riede, Klaus

Rietbroek, Roelof

Righi, Marco

Righini, Gaia

Rignot, Eric

Riihelä, Aku

Rijal, Santosh

Ringerud, Sarah

Rinke, Karsten

Riquelme, Adrián

Riquelme, Adrián J.

Riquelme, Sebastian

Risbøl, Ole

Risojević, Vladimir

Ritter, Tim

Rivalland, Vincent

Rivas Casado, Monica

Riveiro, Belen

Rizkinia, Mia

Rizzoli, Paola

Robert, Odolinski

Roberto, Benedetti

Roberts, Dar

roberts, gareth

Robinson, Nathaniel

Robles, Ramiro

Rocca, Fabio

Rocha, Alfredo

Rocha, Jorge

Rochoux, Mélanie

Rodger, James

Rodning, Chris

Rodrigo Comino, Jesús

Rodrigues, Ana

Rodrigues, João

Rodriguez Gonzalvez, Pablo

Rodriguez Pino, Marcos
Rodriguez Veiga, Pedro

Rodriguez, Antonio

Rodriguez, Dionisio

Rodriguez, Ernesto

Rodriguez, Nemesio

Rodriguez-Caballero, Emilio

Rodriguez-Fernandez, Nemesio

Rodriguez-Morales, Fernando

Rodríguez-Veiga, Pedro

Roelfsema, Chris

Roggero, Marco

Roig, Ignacio Bosch

Rolim, João

Román-Cascón, Carlos

Romano, Filomena

Romanov, Andrey

Romanov, Peter

Romanovskii, Oleg

Romatschke, Ulrike

Romeiser, Roland

Romo Leon, Jose Raul

Roncella, Riccardo

Ronchetti, Giulia

Rong, Li

Rönnholm, Petri

Rosa, Isabel

Roscher, Ribana

Rosi, Ascanio

Rosina, Elisabetta

Rosina, Konstantin

Roth, Guy

Rotman, Stanley

Rott, Helmut

Roueff, Antoine

Roujean, Jean-Louis

Roupioz, Laure

Rouquié, Bastien

Rousset, Baptiste

Roussos, Evangelos

Rovira-Garcia, Adrià

Rowland, Clare

Roy, Aditi

Roy, Alexandre 
Roy, David

Roy, Shouraseni Sen

Royer, Alain

Rucci, Alessio

Rucka, Magdalena

Rudy, Ashley

Ruescas, Ana Belen

Ruhoff, Anderson

Ruhtz, Thomas

Ruiz-Armenteros, Antonio

Ruiz-Etcheverry, Laura

Ruiz-Pérez, Guiomar

Ruotsalainen, Laura

Rupnik, Ewelina

Ruscica, Romina

Russ, John

Russell, Brandon

Rusu, Alexandru

Rusu, Liliana

Rutishauser, Susanne

Rutten, Jantien

Ružić, Igor

Ryan, Jonathan

Rykaczewski, Krzysztof

Ryu, Joo-Hyung

Rzepecka, Zofia

Rzeszewski, Michal

Rzhanov, Yuri

S. Biudes, Marcelo

Sa, Carolina

Sa, Inkyu

Saarinen, Ninni

Sabater, Neus

Saber, Mohamed

Saberioon, Mehdi

Sabol, Donald

Sačkov, Ivan

Sacré, Pierre-Yves

Sadeghi, Morteza

Sadi, Fazle

Sadjadi, Firooz

Saeed, Khalid

Sáenz, Jon
Safanelli, José Lucas

Safont, Gonzalo

Sagan, Vasit

Saha, Auromeet

Saha, Korak

Saha, Mithun

Sahoo, Alok

Sahoo, Sasmita

Said, Faozi

Saito, Hitoshi

Sakai, Tetsu

Sakuno, Yuji

Salagean, Tudor

Salamí, Esther

Salas, Eric

Salas, Eric Ariel

Salata, Stefano

Salazar, Addisson

Saldarriaga-Noreña, Hugo

Salehi, Sara

Salekin, Serajis

Salembier, Philippe

Salini, Reus

Salmon, Brian

Salvaggio, Carl

Salvatori, Rosamaria

Salvi, Maria Cristina

Sam, Lydia

Samat, Alim

Samiappan, Sathishkumar

Samimi-Namin, Kaveh

Sampath, Ruwan

Sampietro, Daniele

Sánchez De Miguel, Alejandro

Sanchez Aparicio, Luis Javier

Sanchez Molina, Jorge Antonio

Sanchez, Juanma Lopez

Sanchez, Nilda

Sánchez-Carnero, Noela

Sánchez-Rodríguez, David

Sanders, Abram

Sankaran, Sindhuja

Sanna, Francesca 
Sano, Edson

Sansosti, Eugenio

Santagati, Cettina

Santamaria-del-Angel, Eduardo

Santarsiero, Giuseppe

Santesteban, Gonzaga

Santi, Emanuele

Santiago, José María

Sanz-Ablanedo, Enoc

Sapucci, Luiz Fernando

Sargent, Isabel

Sarkar, Sudipta

Sarkissian, Alain

Saroli, Michele

Sarrafzadeh, Hossein

Sarría, Francisco Alonso

Sasgen, Ingo

Sathyachandran, Sanath Kumar

Sato, Hisashi

Sato, Motoyuki

Sato, Shinji

Saveliev, Anatoly

Savi, Patrizia

Savin, Igor Yu

Sawada, Yohei

Saylam, Kutalmis

Sazib, Nazmus

Scagliola, Michele

Scaioni, Marco

Scanlon, Bridget

Scannapieco, Antonio Fulvio

Scarnati, Theresa

Scarponi, Daniele

Schabowicz, Krzysztof

Schäfer, Klaus

Schansker, Gert

Scharffenberg, Martin

Scheiber, Rolf

Scheinert, Mirko

Schepers, Dinand

Schepers, James

Scheuchl, Bernd

Schiavulli, Domenico
Schillaci, Calogero

Schindler, Dirk

Schirrmann, Michael

Schizas, Ioannis

Schlaepfer, Daniel

Schlaffer, Stefan

Schleeweis, Karen

Schlund, Michael

Schmalwieser, Alois

Schmidt, Adam

Schmidt, David

Schmihalter, Urs

Schmitt, Andreas

Schmitt, Francois

Schmitt, Micheal

Schneider, Fabian

Schneider, Philipp

Schreck, Carl

Schröder, Dietrich

Schroeder, Dustin

Schubert, Adrian

Schumacher, Johannes

Schuman, Guy

Schuttrumpf, Holger

Schwaab, Jonas

Schwaizer, Gabriele

Schwartz, Craig

Schwarz, Gottfried

Schwarz, Katharina

Schwieger, Anna

Scott, Andrea

Scott, Waymond

Scotti, Roberto

Scudiero, Elia

Seal, Ayan

Sedano, Fernando

Seehaus, Thorsten

Sefercik, Umut

Sefercik, Umut Güneş

Segoni, Samuele

Seidel, Dominik

Seier, Gernot

Selci, Stefano 
Selkowitz, David

Sellami, Akrem

Sellitto, Pasquale

Selva, Massimo

Semedo, Alvaro

Semeraro, Teodoro

Semmling, Maximilian

Sen, Anando

Senay, Gabriel

Senécal, Jean-Francois

Senese, Antonella

Senf, Cornelius

Seo, Bong-Chul

Seo, Eunkyoung

Seoane, Lucia

Sepúlveda, Héctor Hito

Serafino, Francesco

Serbin, Guy

Serio, Carmine

Serra, Yolande

Serrano-Notivoli, Roberto

Serra-Sagristà, Joan

Servin, Manuel

Sessa, Salvatore

Setiyono, Tri

Sevara, Christopher

Seyednasrollah, Bijan

Seyler, Frédérique

Seyoum, Wondwosen

Sfarra, Stefano

Sguerso, Domenico

Shafian, Sanaz

Shah, Rashmi

Shahabfar, Alireza

Shahidi, Reza

Shahtahmassebi, Amirreza

Shahzad, Faisal

Shamseldin, Tamer

Shang, Rong

Shang, Songhao

Shankman, David

Shao, Dangdang

Shao, Zhenfeng
Shapiro, Aurélie

Shariati, Ali

Sharifi, Ehsan

Sharma, Ashish

Sharma, Lakesh

Sharma, Ram

Sharma, Vivek

Shea, Joseph

Sheekela, Baker-Yeboah

Sheffield, Kathryn

Shehaj, Endrit

Shelestov, Andrii

Shen, Huanfeng

Shen, Li

Shendryk, Yuri

Sheppard, Jonathan

Sherwood, Christopher

Sherwood, Steven

Shestakov, Nikolay

Shi, Zhenwei

Shibata, Yuichiro

Shihavuddin, ASM

Shiu, Yi-Shiang

Shixiong, Cao

Shokr, Mohammed

Shore, Jennifer

Short, Naomi

Shoyama, Kikuko

Shrestha, Rupesh

Shuvankar, Ghosh

Sibanda, Mbulisi

Sica, Francescopaolo

Siddique, Muhammad Adnan

Siddiqui, Fasahat Ullah

Sidhu, Nanki

Siegert, Courtney

Sieglaff, Justin

Sieradzki, Rafał

Siewert, Matthias Benjamin

Sifakis, Nicolas

Silva, Carlos Alberto

Silva, Hugo Miguel

Silva, Ivanovitch 


\author{
Silva, Joel \\ Silva-Fuzzo, Daniela \\ Silvestri, Sonia \\ Silvia, Bianchini \\ Sim, Sunhui \\ Simarro, Gonzalo \\ Simeonakis, Elias \\ Simeone, Vincenzo \\ Simic, Anita \\ Simic, Milan \\ Similä, Markku \\ Simis, Stefan \\ Simley, Eric \\ Simmonds, Ian \\ Simms, Daniel \\ Simms, Elizabeth \\ Simoniello, Tiziana \\ Simonsen, Sebastian Bjerregaard \\ Simpson, Jake \\ Sims, Neil \\ Singh, Alka \\ Singh, Gurdeep \\ Singh, Keshav \\ Singh, Kunwar \\ Singh, Ramesh \\ Singh, Sudhir \\ Singha, Suman \\ Sioris, Christopher \\ Sipelgas, Liis \\ Šipoš, Martin \\ Sirmaçek, Beril \\ Sismanidis, Panagiotis \\ Sithole, George \\ Skaggs, Sheldon \\ Skakun, Sergii \\ Skarlatos, Dimitrios \\ Skierucha, Wojciech \\ Skilodimou, Hariklia \\ Skoković, Dražen \\ Skowronek, Sandra \\ Slaton, Michele \\ Sleewaegen, Jean-Marie \\ Slonecker, Terrance
}

Small, Christopher

Small, David

Smallman, Thomas Luke

Snapir, Boris

Snoeij, Paul

Soares Galvao, Lenio

Sobiech-Wolf, Jennifer

Sofia, Giulia

Sohel, Ferdous

Sohn, Hong-Gyoo

Soja-Woźniak, Monika

Sokol, Zbyněk

Sokoletsky, Leonid

Sokos, Efthimios

Sola, Ion

Solander, Kurt

Solano, Francesco

Solano-Correa, Yady Tatiana

Solari, Lorenzo

Solaro, Giuseppe

Solberg, Svein

Soldatenko, Sergei

Solimene, Raffaele

Solla, Mercedes

Solomos, Stavros

Somodi, Imelda

Son, Seunghyun

Sona, Giovanna

Song, Chunqiao

Song, Jie

Song, Jinling

Song, Shalei

Song, Shuli

Song, Woo Jin

Song, Xiaoyu

Song, Yang

Sonnemann, Till

Sonobe, Rei

Soria, Juan

Soriano, Melissa

Soria-Ruiz, Jesus

Sorrentino, Antonio

Sośnica, Krzysztof 
Sothe, Camile

Soulis, Konstantinos

Sourdeval, Odran

Sousa, Joaquim

Spaans, Karsten

Spangehl, Thomas

Sparks, Aaron

Spencer, Michael

Spiliotopoulos, Marios

Spinazze, Andrea

Spyrou, Christos

Sreckovic, Vladimir

Srinivasan, Kathiravan

Srivastava, Sanjeev

Sriver, Ryan

Staal, Arie

Stach, Alfred

Stachlewska, Iwona

Staller, Alejandra

Stamatopoulos, Constantine

Stamnes, Snorre

Stampoulis, Dimitrios

Stancalie, Gheorghe

Stancic, Ivo

Stanic, Samo

Stankovic, Ljubisa

Stapleton, Neil

Starek, Michael

Starosolski, Roman

Stathis, Dimitrios

Stavi, Ilan

Stavn, Robert H.

Stavrakoudis, Dimitris

Stearns, Leigh

Stebel, Kerstin

Steele-Dunne, Susan

Stefan, Ovidiu

Ştefan, Ovidiu

Steffen, Holger

Stehman, Stephen

Stein, Alfred

Stein, Nathan

Steinmeier, Charlotte
Stentoumis, Christos

Stepanov, Oleg

Stephani, Henrike

Stephen, Haroon

Stępniak, Katarzyna

Sterckx, Sindy

Sterenborg, Dick

Stewart, Scott

Stillman, Susan

Stocker, Erich Franz

Stokes, Martin

Stoll, Enrico

Stopa, Justin

Stopforth, Riaan

Storch, Tobias

Stovall, Atticus

Stramska, Malgorzata

Stratoulias, Dimitris

Strigul, Nikolay

Strimbu, Bogdan

Strozzi, Tazio

Strunk, Jacob

Strunz, Günter

Strutton, Peter

Stumpf, Andre

Štych, Přemysl

$\mathrm{Su}$, Hongbo

Su, Tung-Ching

$\mathrm{Su}$, Yanjun

$\mathrm{Su}$, Yuan-Fong

Suarez, Juan

Subirana, Jaume Sanz

Sublime, Jeremie

Suciu, George

Sudakov, Ivan

Sugawara, Hirofumi

Sugimoto, Nobuo

Sugiura, Ryo

Sukhov, Vladimir

Sultana, Rebeka

Sun, Alexander

Sun, Changming

Sun, Jian 


\author{
Sun, Jiguang \\ Sun, Junqiang \\ Sun, KeXun \\ Sun, Liang \\ Sun, Shaojie \\ Sun, Weiwei \\ Sun, Xiaoli \\ Sundar, Kaarthik \\ Sundström, Anu-Maija \\ Suomi, Irene \\ Surl, Luke \\ Surovy, Peter \\ Susaki, Junichi \\ Susanto, R. Dwi \\ Sužiedelytė Visockienė, Jūratė \\ Suzuki, Kazuyoshi \\ Swanson, Dave \\ Swartz, William \\ Swenson, Gary \\ Syed, Abdul Haleem \\ Sykioti, Olga \\ Symeonakis, Elias \\ Szabó, Szilárd \\ Szantoi, Zoltan \\ Szporak-Wasilewska, Sylwia \\ Szulwic, Jakub \\ Szypłowska, Agnieszka \\ Szyszkowicz, Mieczysław \\ Tabatabaeenejad, Alireza \\ Tadayyon, Hadi \\ Taebi, Amirtahà \\ Takano, Yoshihide \\ Takebayashi, Hideki \\ Takewaka, Satoshi \\ Talone, Marco \\ Tammaro, Umberto \\ Tamminga, Aaron \\ Tamura, Masayuki \\ Tan, Jackson \\ Tan, Jing \\ Tan, Kun \\ Tan, Mou Leong \\ Tan, Shurun
}

Tan, Zhou

Tananaev, Nikita

Tang, Hao

Tang, Jingyin

Tang, RongLin

Tang, Wenjun

Tang, Wenwu

Tang, Ying

Tanguy, Maliko

Taniguchi, Kenta

Tao, Xin

Tapete, Deodato

Tapiador, Francisco

Taramelli, Andrea

Tarantino, Cristina

Tarantino, Eufemia

Taravat, Alireza

Tarnavsky, Elena

Tarpanelli, Angelica

Tatsumi, Kenichi

Tauro, Flavia

Tavani, Stefano

Tavenard, Romain

Taylor, John

Tchindjang, Mesmin

Teferle, Norman

Tegowski, Jaroslaw

Teixeira, Adunias Dos Santos

Teixeira, Ricardo

Teja, Kattenborn

Telesca, Vito

Telfer, Matthew

Telling, Jennifer W.

Temerinac-Ott, Maja

Tenedório, José António

Tenenbaum, David Elliot

Tenneson, Karis

Teodoro, Ana Cláudia

Tepe, Emre

Terebes, Romulus

Terefenko, Paweł

Tesei, Alessandra

Tessari, Giulia 
Teunissen, Peter

Thakuri, Sudeep

Thalman, Ryan

Thapa, Rajesh Bahadur

Thau, David

Thayaparan, Thayananthan

Thiel, Michael

Thiery, Yannick

Thomas, Brian F.

Thomas, Jean-Baptiste

Thomas, Nathan Marc

Thompson, Daniel

Thompson, Victor

Thonfeld, Frank

Thorarinsdottir, Ragnheidur

Thordarson, Thor

Tian, Feng

Tian, Jiaojiao

Tian, Yingwei

Ticehurst, Catherine

Tiede, Dirk

Tigkas, Dimitris

Tikhonov, Vasiliy

Tilton, James

Timmermans, Joris

Timothy, Dube

Tings, Bjoern

Tivy, Adrienne

Tobak, Zalán

Tobin, Kenneth

Tochon, Guillaume

Todoroff, Pierre

Tokekar, Pratap

Tokola, Timo

Tolleson, Douglas

Tolomei, Cristiano

Tolpekin, Valentyn

Tomasello, Agostino

Tomaštík, Julián

Tomaszewski, Dariusz

Tomić, Hrvoje

Tommaselli, Antonio

Tong Minh, Dinh Ho
Toniolo, Horacio

Tonkin, Toby

Tooth, Matthew

Torbick, Nathan

Torres, Ricardo Da Silva

Torresan, Chiara

Torres-Perez, Juan

Torres-Rua, Alfonso

Torres-Sanchez, Jorge

Torres-Sospedra, Joaquín

Torti, Emanuele

Tortini, Riccardo

Toscano, Piero

Toschi, Isabella

Tosi, Luigi

Toure, Ally Mounirou

Tournadre, Jean

Toyota, Takenobu

Traganos, Dimos

Tran, Chinh C.

Trasatti, Elisa

Trent, Tim

Treuhaft, Robert

Trier, Øivind Due

Trinder, John

Trivero, Paolo

Troisi, Salvatore

Troncone, Antonello

Tronin, Andrew

Trudel, Melanie

Truong Hong, Linh

Truong-Hong, Linh

Tsagkatakis, Grigorios

Tsai, Chun-Ming

Tsai, HuiPing

Tsai, Yu Hsin

Tsamalis, Christoforos

Tschudi, Mark

Tsendbazar, Nandika

Tseng, Yi-Hsing

Tsinos, Christos

Tsironis, Vasileios

Tsoulis, Dimitrios 
Tudoroiu, Nicolae

Tullis, Jason

Tuomi, Laura

Tuominen, Sakari

Turner, Darren

Turner, David

Tuttle, Samuel

Tuttle, Samuel E.

Twardowski, Michael

Tyralis, Hristos

Tysiąc, Paweł

Tyystjärvi, Esa

Tzanatos, Evangelos

Tzeng, Yu-Chang

Tziavos, Ilias

Udelhoven, Thomas

Udovydchenkov, Ilya

Uezato, Tatsumi

Uiboupin, Rivo

Ulaby, Fawwaz

Ullmann, Tobias

Ulrich, Mathias

Uotila, Petteri

Uprety, Sirish

Urai, Minoru

Urban, Otmar

Urban, Thomas

Urbanski, Shawn

Urdea, Petru

Usman, Muhammad

Usui, Norihisa

Uttieri, Marco

Uzkent, Burak

Vacca, Giuseppina

Vaglio Laurin, Gaia

Vain, Ants

Vakalopoulou, Maria

Vakhitov, Alexander

Vakkari, Ville

Valença, Jónatas

Valentini, Nico

Valerio Iungo, Giacomo

Vallet, Bruno
Valsesia, Diego

Van Aardt, Jan

Van Der Kwast, Johannes

Van Der Merwe, Deon

Van Der Werff, Harald

Van Der Zande, Dimitry

Van Deventer, Heidi

Van Diedenhoven, Bastiaan

Van Kessel, Thijs

Van Laarhoven, Twan

Van Malderen, Roeland

Van Stan, John

Van Wesemael, Bas

Van Wittenberghe, Shari

Van Wychen, Wesley

Vandebroek, Elena

Vanderbilt, Vern

Vanderwel, Mark

Vanhellemont, Quinten

Vanselow, Kim

Vant-Hull, Brian

Vantrepotte, Vincent

Vaquero-Martinez, Javier

Varco, Jac Jeremier

Varnai, Tamas

Varotsos, Costas

Vašát, Radim

Vasilakos, Christos

Vasiliades, Lampros

Vasilkov, Alexander

Vaughn, Nicholas

Vauhkonen, Jari

Vazquez, Jorge

Vega-Garcia, Cristina

Veganzones, Miguel

Veganzones, Miguel Angel

Velázquez, Javier

Velázquez, Ramiro

Velotto, Domenico

Venafra, Sara

Venuti, Giovanna

Verbree, Edward

Verbyla, Dave 
Verde, Simona

Verdú, Antonio Ruiz

Vergos, George

Verhelst, Jolien

Verhoef, Anton

Verhoef, Wouter

Verhoeven, Geert

Verrelst, Jochem

Verstraeten, Willem

Vespe, Francesco

Vettore, Antonio

Via, Stephen

Viana, Helder

Vibhute, Amol D.

Vicent Servera, Jorge

Victoria, Daniel De Castro

Vignola, Frank

Vignudelli, Stefano

Vijay, Saurabh

Vila, Daniel

Vilar, Lara

Vilnrotter, Victor

Vincent, Gregoire

Vincent, Robert

Vincze, Markus

Vinukollu, Raghuveer

Virdis, Salvatore

Virlet, Nicolas

Vishwakarma, Bramha Dutt

Visser, Fleur

Vítek, Stanislav

Vittucci, Cristina

Vivone, Gemine

Vizzari, Marco

Vo, Phong

Vogelzang, Jur

Voight, Carly

Voogt, James

Vox, Giuliano

Vozel, Benoit

Vreugdenhil, Mariette

Vrieling, Anton

Vulic, Milivoj
Vuolo, Francesco

Wackerman, Christopher

Wada, Akiyoshi

Wagenbrenner, Natalie

Wagle, Pradeep

Wagner, Simon A

Walawender, Jakub

Walczykowski, Piotr

Waldhoff, Guido

Waldteufel, Philippe

Walega, Andrzej

Walker, Nick

Wallace, Luke

Walter, Thomas

Walter-Shea, Elizabeth

Walther, Sophia

Wan, Jia

Wan, Zhanming

Wang, Bin

Wang, Chao

Wang, Chenxi

Wang, Chi-Kuei

Wang, Chuyuan

Wang, Di

Wang, Fu-Kang

Wang, Guifen

Wang, Guochao

Wang, Haihong

Wang, Hao

Wang, Heshun

Wang, Hongquan

Wang, Hsueh-Ching

Wang, Jiao

Wang, Jida

Wang, Jinfeng

Wang, Jinhu

Wang, Jun

Wang, Kan

Wang, Kang

Wang, Mingshu

Wang, Panshi

Wang, Pei

Wang, Pichao 


\author{
Wang, Ran \\ Wang, Teng \\ Wang, Tiejun \\ Wang, Weile \\ Wang, Wenhui \\ Wang, Xianwei \\ Wang, Xuan \\ Wang, Xuantong \\ Wang, Yi \\ Wang, Yong \\ Wang, Yonghui \\ Wang, Yu \\ Wang, Yujie \\ Wang, Yunsheng \\ Wang, Zhihui \\ Wang, Zhuosen \\ Wanik, David \\ Warner, Roland \\ Watkins, James \\ Watson, Cameron Scott \\ Watson, Dennis \\ Watson, Des \\ Watson, Elizabeth Burke \\ Way, Robert \\ Wayand, Nicholas \\ Weber, Markus \\ Weber, Robert \\ Wegner, Jan \\ Wei, Anhua \\ Wei, Jianwei \\ Weidinger, Tamas \\ Weinmann, Martin \\ Weinmann, Michael \\ Weiskittel, Aaron \\ Weissgerber, Flora \\ Welc, Fabian \\ Wen, Jianguang \\ Wen, Yangmao \\ Wendleder, Anna \\ Wendt, Anja \\ Wenzel, Susanne \\ Werle, Dirk \\ Wernerv, Piotr
}

Wersing, Heiko

Westerdahl, Dane

Westerhoff, Rogier

Westerlund, Tomi

Whelan, Chad

White, Devin

White, Lori

White, Scott

Wicaksono, Pramaditya

Wick, Gary

Wiedemann, Kenia

Wiegner, Matthias

Wiehle, Stefan

Wieland, Marc

Wielgosz, Paweł Aleksander

Wierzbicki, Damian

Wilbert, Stefan

Wildmann, Norman

Wiley, Robert

Wilken, Dennis

Wilkes, Phil

Wilkes, Thomas C

Wilkin, John

Wilkinson, Ben

Willem, J.D. van Leeuwen

Williams, Gustavious Paul

Williams, Simon

Williamson, Grant

Williamson, Scott

Willmes, Sacha

Wilson, Anna

Wilson, Cara

Wilusz, Daniel

Wimberly, Michael

Wimmers, Anthony

Witmer, Frank

Witschas, Benjamin

Wohlfart, Christian

Wolny, Jennifer

Wolters, Erwin

Won, Chee Sun

Won, Joong-Sun

Wong, Frankie Kwan Kit 
Woo, Hyenkyun

Wood, Kimberly

Woods, Murray

Woolway, Richard

Woolway, Richard Iestyn

Worqlul, Abeyou

Wright, Christopher

Wright, Logan

Wright, William

$\mathrm{Wu}, \mathrm{Bin}$

$\mathrm{Wu}, \mathrm{Bo}$

Wu, Changshan

$\mathrm{Wu}$, Chih-Da

$\mathrm{Wu}$, Fan

Wu, Guofeng

$\mathrm{Wu}$, Qiusheng

$\mathrm{Wu}$, Yonghua

$\mathrm{Wu}$, Yongsheng

Wulder, Michael

Wylie, Bruce

Xavier, Antonio

Xi, Baike

Xia, Jianyang

Xian, George

Xiang, Deliang

Xiao, Pengfeng

Xiao, Wen

Xie, Jiping

Xie, Yingying

Xie, Zhong

Xie, Zunyi

Xin, Qinchuan

Xing, Cheng

Xiong, Jun

Xiong, Siting

$\mathrm{Xu}$, Chong

$\mathrm{Xu}$, Jian

$X u$, Lijun

$\mathrm{Xu}, \mathrm{Lu}$

$\mathrm{Xu}$, Qing

$\mathrm{Xu}$, Xiaobiao

$\mathrm{Xu}$, Xiaoguang

$\mathrm{Xu}$, Xiaohua
Xu, Xiaolan

$\mathrm{Xu}$, Xiaoming

$\mathrm{Xu}, \mathrm{Xin}$

$\mathrm{Xu}$, Yongming

$\mathrm{Xu}$, Yuyue

XU., H.

Yackel, John

Yadav, Kamini

Yagüe-Martinez, Nestor

Yajima, Taro

YAMAGUCHI, Yasushi

Yamamoto, Hirokazu

Yamamoto, Naomichi

Yamanaka, Goro

Yamazaki, Fumio

Yan, Dong

Yan, Hongxiang

Yan, Kai

Yan, Lin

Yan, Waiyeung

Yan, Xiao-Hai

Yan, Yingwei

Yando, Erik

Yang, Chan-Su

Yang, Chenghai

Yang, Dingtian

Yang, Jason

Yang, Jinn-Min

Yang, John Xun

Yang, John Xun

Yang, Kang

Yang, Liping

Yang, Long

Yang, Ming-Der

Yang, Peiqi

Yang, Ping

Yang, Shu-Chih

Yang, Song

Yang, Wei

Yang, Wen

Yang, Wenze

Yang, Xi

Yang, Xiaofeng 


\begin{tabular}{|c|c|}
\hline Yang, Yang & Yurganov, Leonid \\
\hline Yang, Zhijing & Yurovsky, Yury Yu \\
\hline Yang, Zutao & Yuzugullu, Onur \\
\hline Yanovsky, Igor & Zabalza, Jaime \\
\hline Yao, Tian & Zagajewski, Bogdan \\
\hline Yavariabdi, Amir & Zaikin, Oleg Sergeevich \\
\hline Yazdani, Javad & Zak, Andrzej \\
\hline Yearsley, John & Zakharov, Igor \\
\hline Yee, Leung & Zakšek, Klemen \\
\hline Yeh, Sheng Lih & Zaman, Bushra \\
\hline Yen, Tianming & Zambrano, Francisco \\
\hline Yesou, Herve & Zaminpardaz, Safoora \\
\hline Yetemen, Omer & Zaron, Edward \\
\hline Yi, Bingqi & Zatelli, Paolo \\
\hline Yi, Shuang & Zawadzki, Jarosław \\
\hline Yin, Bangsheng & Zazo, Santiago \\
\hline Yin, Jifu & Zehtabian, Amin \\
\hline Yin, Zun & Zeleňáková, Martina \\
\hline Ylänne, Henni & Zema, Demetrio Antonio \\
\hline Yoon, Yoke & Zemke, Julian \\
\hline Yoshino Watanabe, Fernanda Sayuri & Zeng, Jiangyuan \\
\hline Yoshioka, Hiroki & Zeng, Yelu \\
\hline You, Cheol Hwan & Zeng, Yijian \\
\hline You, Xinge & Zeraatpisheh, Mojtaba \\
\hline You, Yalei & Zewdie, Worku \\
\hline Young, Alisa & Zhai, Pengwang \\
\hline Young, Matthew & Zhan, Wenfeng \\
\hline Young, Stephen & Zhan, Xiwu \\
\hline Yu, Kang & Zhang, Ce \\
\hline Yu, Ting-To & Zhang, Chao \\
\hline Yu, Xiaolei & Zhang, Chuanrong \\
\hline Yu, Xiaolong & Zhang, Guoqing \\
\hline Yu, Xiaowei & Zhang, Guosheng \\
\hline Yu, Yifan & Zhang, Hankui \\
\hline Yu, Yunyue & Zhang, Hao \\
\hline Yu, Zhen & Zhang, Haopeng \\
\hline Yuan, Fei & Zhang, Hong \\
\hline Yuan, Hua & Zhang, Hua \\
\hline yuan, qiangqiang & Zhang, Huihui \\
\hline Yuan, Zhenghang & Zhang, Jiashu \\
\hline Yue, Peng & Zhang, Jiaxu \\
\hline Yue, Xu & Zhang, Jun \\
\hline Yunta, Felipe & Zhang, Lefei \\
\hline
\end{tabular}




Zhang, Lei
Zhang, Liangpei
Zhang, Libao
Zhang, Liqiang
Zhang, Lu
Zhang, Miao
Zhang, Minwei
Zhang, Qilin
Zhang, Qun
Zhang, Shuai
Zhang, Su
Zhang, Wen
Zhang, Xi
Zhang, Xianfeng
Zhang, Yan
Zhang, Yao
Zhang, Yong-Fei
Zhang, Yongguang
Zhang, Yu
Zhang, Yuanchong
Zhang, Yuanzhi
Zhang, Yudong
Zhang, Yunsheng
Zhang, Zhou
Zhao, Chaoying
Zhao, Feng
Zhao, Lijun
Zhao, Long
Zhao, Meng
Zhao, Tianjie
Zhao, Wei
Zhao', Ying
Zheng, Gang
Zheng, Yuanjin
Zhizhin, Mikhail
Zhong, Liheng
Zhong, Ruofei
Zanfei
Zuoqing
Zhoug
Zho

Zhou, Hui

Zhou, Ji

Zhou, Jun

Zhou, Qiang

Zhou, Song

Zhou, Tian

Zhou, Xiaochi

Zhou, Xiran

Zhou, Yuan

Zhou, Yuyu

Zhu, Dan

Zhu, Feng

Zhu, Kai

Zhu, Lei

Zhu, Lingxiao

Zhu, Qing

Zhu, Weining

Zhu, Zaichun

Zhu, Zhe

Zhuo, Xiangyu

Ziemann, Amanda

Zimmermann, Robert

Zingaretti, Primo

Ziniti, Beth

Zinner, Tobias

Zivcak, Marek

Zlinszky, András

Zoffoli, Maria Laura

Zorzi, Mattia

Zou, Lidong

Zribi, Mehrez

Zsedrovits, Tamás

Zucca, Francesco

Zuo, Boxin

Zus, Florian

Zweig, Christa

Zweiri, Yahya

Zwieback, Simon

Zygielbaum, Arthur

(C) 2019 by the authors. Licensee MDPI, Basel, Switzerland. This article is an open access article distributed under the terms and conditions of the Creative Commons Attribution (CC BY) license (http://creativecommons.org/licenses/by/4.0/). 